\title{
Modeling Transient Heat Conduction and Radiative Transport in Semitransparent Media: A Tool for the Interpretation of Reflectivity Data
}

\author{
Manuela Musella • Hans Rudolf Tschudi
}

Received: 23 December 2005 / Accepted: 16 November 2007 / Published online: 18 December 2007

(C) Springer Science+Business Media, LLC 2007

\begin{abstract}
In this work the problem of interpretation of reflectivity data for semitransparent materials at high temperatures is addressed. A detailed analysis of the transient thermal response of a participating medium subjected to a pulse of finite energy is performed using a new method developed to solve the general equation of energy transfer for a conductive, absorbing, emitting, and scattering medium. The model, previously presented for a material with constant optical properties (Musella, Tschudi, Int J Thermophys 26:981, 2005), has been upgraded to encompass a much wider scenario where the thermophysical and optical properties are temperature dependent. This allows the study of the transient reflectivity of laser-heated samples where high heating rates and strong temperature gradients occur near the surface. Considerable differences of the reflectivity values for the same surface temperature calculated in the heating and cooling phases, both different from the corresponding values for an isothermal sample, are reported in accordance with experimental results.
\end{abstract}

Keywords Ceramics $\cdot$ Emissivity $\cdot$ High temperature $\cdot$ Pyrometry ·

Radiative transport

\section{Introduction}

The significance of radiative properties as process data for technical facilities has increased during recent years motivated by a constant demand from industry. A subject

\footnotetext{
M. Musella $\cdot$ H. R. Tschudi ( $\varangle)$

Paul Scherrer Institut, CH-5232 Villigen PSI, Switzerland

e-mail: hansrudolf.tschudi@psi.ch

M. Musella

European Commission Joint Research Centre, Institute for Energy, P.O. Box 2,

NL-1755 ZG Petten, The Netherlands

e-mail: manuela.musella@jrc.nl
} 
of particular interest among engineers and materials physicists is the knowledge of radiative properties for semitransparent media (STM) at high temperatures.

The application domain is broad. Nowadays, ceramic materials, a typical example of STM, are in fact widely employed in components for high-temperature use. As a result of their corrosion-resistant and insulation properties, they are, for instance, used as thermal barrier coatings in advanced turbine engines, space vehicles, and chemical and nuclear reactors. In all these applications, the calculation of energy balances, energy losses, and temperature gradients in the material is of fundamental importance.

In order to describe the internal energy transport of STM, the radiative properties of the material, such as the absorption and scattering coefficients, and their temperature dependences must be known. These properties are determined from reflectivity and transmission measurements which ideally should be performed under isothermal conditions. The high temperatures required to characterize STM, however, can be only attained with transient heating of the sample, using, for instance, a laser. Laser heating causes strong temperature gradients in the sample, and appreciable temperature differences may occur within the optical escape depth of the material. Since, in general, the radiative properties of STM clearly depend on temperature, a strong temperature gradient within the optical escape depth has the effect that regions with different absorption and scattering properties contribute to the reflected light, and the relationship between the measured reflectivity and the radiative properties of the material becomes complex. For connecting measured reflectivity and transmission data with the radiative properties, detailed modeling of the interaction of light with matter and of the combined effects of radiative and conductive heat transfer is therefore required. Moreover, since most of the measurement techniques are based on dynamic records, as pointed out, the thermal transient behavior has to be analyzed.

The topic of radiation effects on transient energy transfer is of large interest and has been extensively studied during the last 50 years [1,2]. Unfortunately, most of the studies are focused on very special cases because of the mathematical complexity of the problem. The topic of transient combined conduction and radiative transfer is even less studied, and only very particular problems like the absence of scattering [3-6], extremely short times $[7,8]$, or very specific applications $[9,10]$ have been addressed. The analysis of more general cases proved to be quite complex with long and tedious procedures for their numerical solution $[11,12]$.

To deal with this problem, we proposed in a previous study [13] a new method for solving the heat conduction and radiation transfer equations that is general and fast from the computational point of view. The central idea is to express the solution of the two-flux equations for radiation $[14,15]$ as the sum of two contributions, namely, of an approximate special solution of the full, inhomogeneous two-flux equations accounting for absorption, scattering, and emission of radiation and of a solution of the homogeneous equations, the equations without the emission term. The special solution is dominant in the interior of the participating medium and properly accounts for the thermal emission term but does not recognize, in general, the boundary conditions for the radiation field while the second contribution, important in a surface layer, is used to match these boundary conditions. The radiative flow originating from the approximate special solution corresponds to the diffusion approximation $[14,16]$. This flow is completed in the surface region by a radiative flow contribution due to the 
solution of the homogeneous equations. In [13], these ideas are applied to the problem of an infinite slab of gray material. An explicit expression for the special solution of the inhomogeneous equations is derived in terms of the temperature field, and an analytical solution for the homogeneous equation is given under the assumption that the optical properties of the material are constant in the surface layer.

In this paper we extend the analysis to the case where the thermophysical and optical material parameters are true temperature functions everywhere in the layer. A method is developed to calculate the solution of the homogeneous equations and the reflectivity of the slab, taking into account the temperature dependence of the optical properties and the actual temperature profile inside the sample. In addition, a formula is derived for the transient temperature that a one-color pyrometer would measure using the actual transient emissivity values. It is shown that this temperature corresponds to the actual surface temperature for an opaque sample although the radiation probed with the pyrometer originates from a surface layer with a possibly non-uniform temperature.

Based on these results, a numerical algorithm is developed and implemented as a $\mathrm{C}++$ code. This code is used to study the behavior of a layer of yttria-stabilized zirconia irradiated with a laser pulse. The temperature profile of the sample is studied as a function of the key parameters, and its influence on the transient reflectivity of the layer is investigated. The results obtained are then compared with experimental investigations found in the literature.

\section{Physical Model and Governing Equations}

\subsection{Heat Conduction and Radiative Transfer in a Slab}

Combined heat conduction and radiative transport in a slab consisting of a homogeneous absorbing, scattering, and emitting medium is considered. The material properties may depend on temperature. If the temperature and the energy fluxes vary only in the direction perpendicular to the surface of the slab, the $x$-direction, the energy equation is given by

$$
d(T) c_{p}(T) \frac{\partial T(x, t)}{\partial t}=-\frac{\partial}{\partial x}\left(J_{Q}+J\right)
$$

where $d(T)$ denotes the density of the material and $c_{p}(T)$ the specific heat per mass. Equation 1 must be supplied with expressions for the conductive and radiative fluxes, $J_{Q}$ and $J$. The conductive flux $J_{Q}$ is given by Fourier's equation,

$$
J_{Q}=-k(T) \frac{\partial T(x, t)}{\partial x}
$$

If the physical properties vary only in the direction perpendicular to the surface of the slab, an expression for the radiative flux $J$ is appropriately given using the two-flux approximation [14]. In this case the description of the radiative field is reduced to two radiant fluxes, $J_{+}$and $J_{-}$, in the positive and negative $x$-directions (see Fig. 1). With 


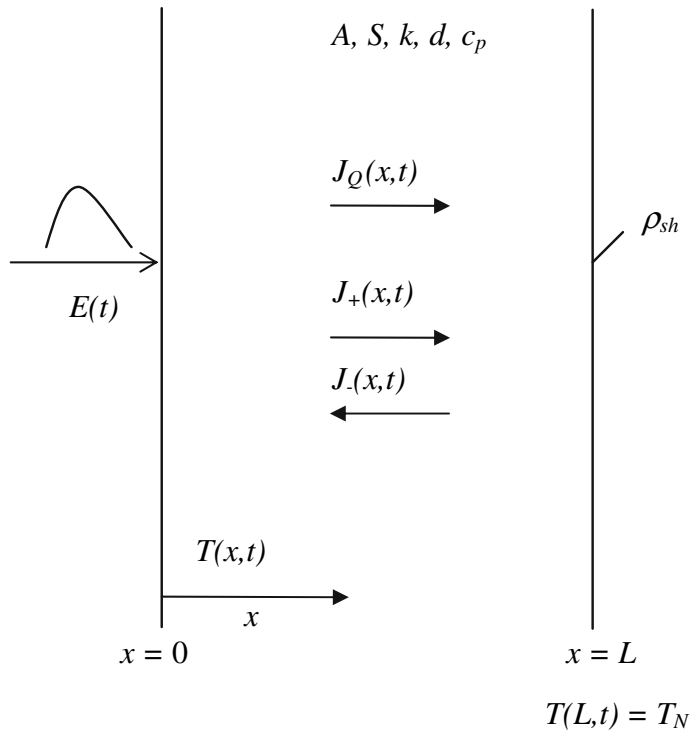

Fig. 1 Geometry, coordinate system, and nomenclature of the studied physical system

the Kubelka-Munk notation [15], the two fluxes, $J_{+}$and $J_{-}$, are expressed by the total radiative flux $J$ and a second quantity $F$ which is proportional to the local energy density of the radiative field:

$$
\begin{aligned}
& J(x, t)=J_{+}(x, t)-J_{-}(x, t) \\
& F(x, t)=J_{+}(x, t)+J_{-}(x, t)
\end{aligned}
$$

For these quantities, the differential equations,

$$
\frac{\partial J(x, t)}{\partial x}=-A\left[F(x, t)-2 \sigma_{\mathrm{B}} T^{4}\right]
$$

and

$$
\frac{\partial F(x, t)}{\partial x}=-(A+2 S) J(x, t)
$$

are valid if the absorption coefficient $A$ and the scattering coefficient $S$ do not depend on the wavelength (gray body).

It was shown in a previous paper [13] that the solution of the system of Eq. 3 can be expressed as a sum of two contributions:

$$
\begin{gathered}
J=J^{\mathrm{si}}+J^{\mathrm{h}} \\
F=F^{\mathrm{si}}+F^{\mathrm{h}}
\end{gathered}
$$

where $J^{\text {si }}$ and $F^{\text {si }}$ represent a special solution of the full equations, inhomogeneous with respect to the radiation quantities $J$ and $F$, and $J^{\mathrm{h}}$ and $F^{\mathrm{h}}$ represent the solu- 
tion of the homogeneous equations of radiative transfer without the emission term $2 A \sigma_{\mathrm{B}} T^{4}$. This solution is needed to match the boundary conditions for the radiative field. An approximation to the special solution of the full, inhomogeneous Eqs. 3a and $3 \mathrm{~b}$ is given by [13]

$$
F^{\mathrm{si}}=2 \sigma_{\mathrm{B}} T^{4}
$$

and

$$
J^{\mathrm{si}}=-\frac{8 \sigma_{\mathrm{B}} T^{3}}{A+2 S} \frac{\partial T}{\partial x}
$$

The expressions in Eq. 5 are the first terms of a recursive solution of the two-flux Eq. 3. Equation $5 b$ corresponds to the diffusion approximation [16,14].

Substituting Eq. 4a in Eq. 1 yields

$$
d(T) c_{p}(T) \frac{\partial T(x, t)}{\partial t}=-\frac{\partial}{\partial x}\left(J_{Q}+J^{\mathrm{si}}\right)-\frac{\partial J^{\mathrm{h}}}{\partial x} .
$$

With Eqs. 2 and $5 b$ the transient energy equation can, thus, be rewritten as

$$
d(T) c_{p}(T) \frac{\partial T(x, t)}{\partial t}=-\frac{\partial J^{\prime}}{\partial x}-\frac{\partial J^{\mathrm{h}}}{\partial x}
$$

where

$$
J^{\prime}=-k_{\mathrm{hrt}}(T) \frac{\partial T(x, t)}{\partial x}
$$

with

$$
k_{\mathrm{hrt}}(T)=k(T)+\frac{8 \sigma_{\mathrm{B}}}{A+2 S} T^{3}
$$

Equation 6 has now the form of the classical transient energy equation where the first term on the right side is analogous to Fourier's equation (with $k_{\text {hrt }}$ describing the combined effects of heat conduction and radiative transport) and the second term $\frac{\partial J^{\mathrm{h}}}{\partial x}$ represents an additional outer heat source.

\subsubsection{Solution of the Homogeneous Two-Flux Equations}

In order to find a general expression for the term $\frac{\partial J^{\mathrm{h}}}{\partial x}$ in Eq. 6, the homogeneous two-flux equations, Eqs. $3 \mathrm{a}$ and $\mathrm{b}$ without the emission term, have to be solved. These equations can be analytically solved if the absorption and scattering coefficients do 


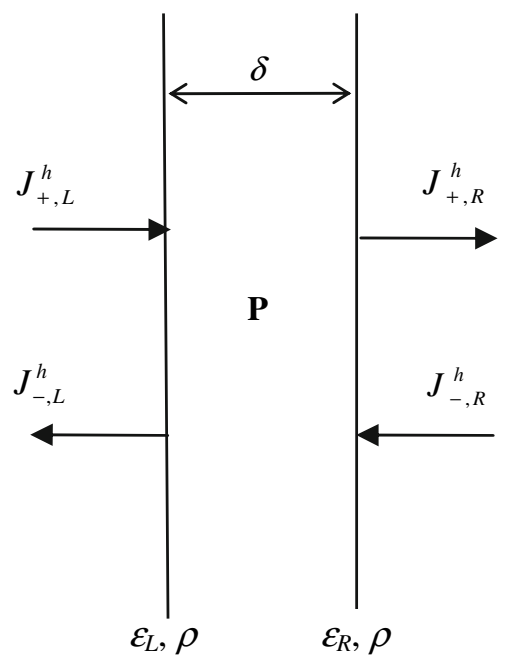

Fig. 2 Radiative flows for a layer of thickness $\delta$. The homogeneous two-flux equations for radiative transfer connect the flows on the left-hand side of the layer with the flows on the right-hand side through the transfer matrix $\mathbf{P}$

not vary with $x$. In this case, the solution can be written with a propagation matrix which has the form (see Fig. 2),

$$
\left(\begin{array}{c}
J_{+, \mathrm{R}}^{\mathrm{h}} \\
J_{-, \mathrm{R}}^{\mathrm{h}}
\end{array}\right)=\mathbf{P}\left(\begin{array}{c}
J_{+, \mathrm{L}}^{\mathrm{h}} \\
J_{-, \mathrm{L}}^{\mathrm{h}}
\end{array}\right)
$$

where $J_{ \pm, \mathrm{L}}^{\mathrm{h}}$ and $J_{\mp, \mathrm{R}}^{\mathrm{h}}$ represent the in- and out-going fluxes at the left and right sides of a layer of thickness $\delta$ and

$$
\mathbf{P}=\left(\begin{array}{ll}
P_{11} & P_{12} \\
P_{21} & P_{22}
\end{array}\right)
$$

is the propagation matrix, the elements of which are equal to

$$
\begin{aligned}
& P_{11}=\cosh (W \delta)-\frac{A+S}{W} \sinh (W \delta) \\
& P_{12}=-P_{21}=\frac{S}{W} \sinh (W \delta) \\
& P_{22}=\cosh (W \delta)+\frac{A+S}{W} \sinh (W \delta)
\end{aligned}
$$

with $W=\sqrt{A^{2}+2 A S}$. It follows that $\operatorname{det} \mathbf{P}=P_{11} P_{22}-P_{12} P_{21}=1$.

If an inhomogeneous layer is considered with spatially varying absorption and scattering coefficients, a solution can be found by subdividing the whole layer into a 
system of thin layers, each having constant material properties. In this case the propagation matrix of the whole layer is the matrix product of the matrices of the single sublayers (see Sect. 2.2).

If the incident fluxes, $J_{+, \mathrm{L}}^{\mathrm{h}}$ and $J_{-, \mathrm{R}}^{\mathrm{h}}$, on either side of a layer are given, an alternative formulation of the solution of the homogeneous two-flux equations is appropriate. The outgoing fluxes, $J_{-, \mathrm{L}}^{\mathrm{h}}$ and $J_{+, \mathrm{R}}^{\mathrm{h}}$, can then be expressed as linear combinations of the two impinging ones,

$$
\begin{aligned}
& J_{-, \mathrm{L}}^{\mathrm{h}}=\rho_{\mathrm{L}} J_{+, \mathrm{L}}^{\mathrm{h}}+\tau_{\mathrm{R}} J_{-, \mathrm{R}}^{\mathrm{h}} \\
& J_{+, \mathrm{R}}^{\mathrm{h}}=\tau_{\mathrm{L}} J_{+, \mathrm{L}}^{\mathrm{h}}+\rho_{\mathrm{R}} J_{-, \mathrm{R}}^{\mathrm{h}}
\end{aligned}
$$

where the reflectivities $\rho_{\mathrm{L}}$ and $\rho_{\mathrm{R}}$ and the transmissivities $\tau_{\mathrm{L}}$ and $\tau_{\mathrm{R}}$ of the layer all have values in the interval $[0,1]$. These four quantities can be expressed by the elements of the propagation matrix. If $J_{-, \mathrm{R}}^{\mathrm{h}}=0$, then

$$
\rho_{\mathrm{L}}=\frac{J_{-, \mathrm{L}}^{\mathrm{h}}}{J_{+, \mathrm{L}}^{\mathrm{h}}}
$$

and

$$
\tau_{\mathrm{L}}=\frac{J_{+, \mathrm{R}}^{\mathrm{h}}}{J_{+, \mathrm{L}}^{\mathrm{h}}}
$$

yielding

$$
\rho_{\mathrm{L}}=-\frac{P_{21}}{P_{22}}
$$

and

$$
\tau_{\mathrm{L}}=\frac{1}{P_{22}}
$$

Similarly, if $J_{+, \mathrm{L}}^{\mathrm{h}}=0$, then

$$
\rho_{\mathrm{R}}=\frac{J_{+, \mathrm{R}}^{\mathrm{h}}}{J_{-, \mathrm{R}}^{\mathrm{h}}}
$$

and

$$
\tau_{\mathrm{R}}=\frac{J_{-, \mathrm{L}}^{\mathrm{h}}}{J_{-, \mathrm{R}}^{\mathrm{h}}}
$$


yielding

$$
\rho_{\mathrm{R}}=\frac{P_{12}}{P_{22}}
$$

and

$$
\tau_{\mathrm{R}}=\frac{1}{P_{22}}=\tau_{\mathrm{L}}=\tau_{\delta}
$$

On the other hand, the matrix elements $P_{11}, P_{12}, P_{21}, P_{22}$ may be also expressed by $\rho_{\mathrm{L}}, \rho_{\mathrm{R}}$, and $\tau_{\delta}$ as

$$
\begin{aligned}
& P_{12}=\frac{\rho_{\mathrm{L}}}{\tau_{\delta}} \\
& P_{21}=-\frac{\rho_{\mathrm{R}}}{\tau_{\delta}} \\
& P_{22}=\frac{1}{\tau_{\delta}} \\
& P_{11}=\frac{1}{P_{22}}\left(1+P_{12} P_{21}\right)=\frac{1}{\tau_{\delta}}\left(\tau_{\delta}^{2}-\rho_{\mathrm{L}} \rho_{\mathrm{R}}\right)
\end{aligned}
$$

The power flow $\Delta J^{\mathrm{h}}$ absorbed in the layer as a function of the impinging fluxes is given by

$$
\Delta J^{\mathrm{h}}=J_{\mathrm{L}}^{\mathrm{h}}-J_{\mathrm{R}}^{\mathrm{h}}=J_{+, \mathrm{L}}^{\mathrm{h}}-J_{-, \mathrm{L}}^{\mathrm{h}}+J_{-, \mathrm{R}}^{\mathrm{h}}-J_{+, \mathrm{R}}^{\mathrm{h}}
$$

which together with Eqs. 10a and b gives

$$
\Delta J^{\mathrm{h}}=\varepsilon_{\mathrm{L}} J_{+, \mathrm{L}}^{\mathrm{h}}+\varepsilon_{\mathrm{R}} J_{-, \mathrm{R}}^{\mathrm{h}}
$$

with

$$
\varepsilon_{\mathrm{L} / \mathrm{R}}=1-\rho_{\mathrm{L} / \mathrm{R}}-\tau_{\delta}
$$

\subsection{Solution for a Slab Subjected to Laser Heating}

\subsubsection{Discretization of the Layer}

We consider an infinitely extended plane layer of thickness $L$ initially at temperature $T_{N}$ and placed in vacuum. The slab is uniformly irradiated on the front surface by a laser pulse of finite width while the rear surface is kept at constant temperature $T_{N}$ (see Fig. 1). The layer is assumed to be homogeneous with physical properties that may depend on temperature. The absorption and scattering coefficients do not depend on wavelength (gray body). The transient energy equation representing the system is then given by Eq. 6 . 
For the numerical solution, the investigated slab is divided into $N$ cells indexed with $n=1,2, \ldots, N$. Each cell of thickness $\delta_{n}$ is considered to be isothermal with temperature $T_{n}$ and with constant physical properties. The discrete analogous of Eq. 6 for this case is (see [13], Sect. 3)

$$
\frac{\mathrm{d} T_{n}}{\mathrm{~d} t}=\frac{1}{\left(d c_{p}\right)_{n}} \frac{1}{\delta_{n}}\left(J_{n-1}^{\prime}-J_{n}^{\prime}\right)+\frac{1}{\left(d c_{p}\right)_{n}} \frac{1}{\delta_{n}} \Delta J_{n}^{\mathrm{h}}
$$

where

$$
J_{n}^{\prime}=-2 \frac{k_{\mathrm{hrt}, n} k_{\mathrm{hrt}, n+1}}{\delta_{n} k_{\mathrm{hrt}, n+1}+\delta_{n+1} k_{\mathrm{hrt}, n}}\left(T_{n+1}-T_{n}\right)
$$

and $\Delta J_{n}^{\mathrm{h}}$ is given by Eq. 14. If three consecutive layers have the same thickness, $\delta_{n-1}=\delta_{n}=\delta_{n+1}$, the derivative $\frac{\mathrm{d} T_{n}}{\mathrm{~d} t}$ is equal to the value of the partial derivative $\frac{\partial T(x, t)}{\partial t}$, given by Eq. 6 , at the middle of the layer $n$ up to a term of the order of $\delta_{n}^{2}$. Otherwise, additional deviations of the orders $\delta_{n}-\delta_{n-1}, \delta_{n}-\delta_{n+1}$ occur.

\subsubsection{Evaluation of the Source Term of Eq. 16}

In order to obtain a closed system of equations, the second term on the right-hand side of Eq. 16 must be expressed as a function of the temperature $T_{n}$ and the boundary conditions for temperature and radiative flows on both sides of the slab. First, one observes that the power absorbed in the layer $n$ is given by

$$
\Delta J_{n}^{\mathrm{h}}=\varepsilon_{n}\left(J_{+, n-1}^{\mathrm{h}}+J_{-, n}^{\mathrm{h}}\right)
$$

with

$$
\varepsilon_{n}=1-\frac{P_{12}^{(n)}+1}{P_{22}^{(n)}}
$$

Equation 14 has been used and the fact that each individual layer is homogeneous implies $P_{21}^{(n)}=-P_{12}^{(n)}$ and, thus, $\varepsilon_{\mathrm{L}}^{(n)}=\varepsilon_{\mathrm{R}}^{(n)}=\varepsilon_{n}$.

As a second step, the radiative fluxes, $J_{-, n}^{\mathrm{h}}$ and $J_{+, n}^{\mathrm{h}}$, are expressed by the corresponding fluxes at the boundaries of the slab. For that purpose, we divide the cell structure of the slab into two substructures, a left one grouping the cells from 0 to $n$ and a right one grouping the cells from $n+1$ to $N$ (see Fig. 3). Since the propagation matrix for a sequence of adjacent layers is the product of the individual propagation matrices $\mathbf{P}^{(n)}$ for the single layers, the propagation matrix $\mathbf{L}_{n}$ of the left substructure is given by

$$
\mathbf{L}_{n}=\mathbf{P}^{(1)} \mathbf{P}^{(2)} \ldots \mathbf{P}^{(n)}
$$




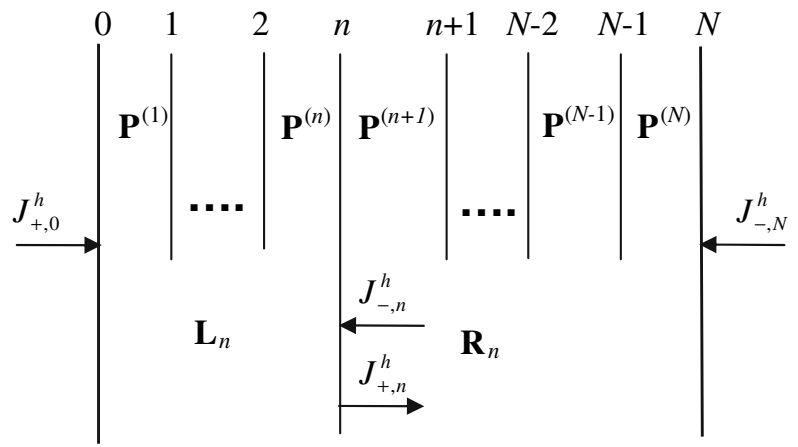

Fig. 3 Subdivision of the slab into $N$ layers used for the numerical implementation (for details, see text)

and the propagation matrix $\mathbf{R}_{n}$ for the right substructure by

$$
\mathbf{R}_{n}=\mathbf{P}^{(n+1)} \mathbf{P}^{(n+2)} \ldots \mathbf{P}^{(N)}
$$

According to Eq. 10, it is at the boundary $n$

$$
\begin{aligned}
& J_{-, n}^{\mathrm{h}}=\rho_{\mathrm{L}, n}^{\mathrm{R}} J_{+, n}^{\mathrm{h}}+\tau_{n}^{\mathrm{R}} J_{-, N}^{\mathrm{h}} \\
& J_{+, n}^{\mathrm{h}}=\rho_{\mathrm{R}, n}^{\mathrm{L}} J_{-, n}^{\mathrm{h}}+\tau_{n}^{\mathrm{L}} J_{+, 0}^{\mathrm{h}}
\end{aligned}
$$

where $\rho_{\mathrm{L}, n}^{\mathrm{R}}$ and $\tau_{n}^{\mathrm{R}}$ refer to the reflectivity and transmissivity of the right substructure and $\rho_{\mathrm{R}, n}^{\mathrm{L}}$ and $\tau_{n}^{\mathrm{L}}$ to the left substructure. From Eq. 20, the fluxes, $J_{-, n}^{\mathrm{h}}$ and $J_{+, n}^{\mathrm{h}}$, at the boundary $n$ separating the layers $n$ and $n+1$ can be expressed by the fluxes, $J_{+, 0}^{\mathrm{h}}$ and $J_{-, N}^{\mathrm{h}}$, impinging on either side of the whole slab yielding

$$
\begin{aligned}
& J_{+, n}^{\mathrm{h}}=\frac{\tau_{n}^{\mathrm{L}} J_{+, 0}^{\mathrm{h}}+\tau_{n}^{\mathrm{R}} \rho_{\mathrm{R}, n}^{\mathrm{L}} J_{-, N}^{\mathrm{h}}}{1-\rho_{\mathrm{L}, n}^{\mathrm{R}} \rho_{\mathrm{R}, n}^{\mathrm{L}}} \\
& J_{-, n}^{\mathrm{h}}=\frac{\tau_{n}^{\mathrm{R}} J_{-, N}^{\mathrm{h}}+\tau_{n}^{\mathrm{L}} \rho_{\mathrm{L}, n}^{\mathrm{R}} J_{+, 0}^{\mathrm{h}}}{1-\rho_{\mathrm{L}, n}^{\mathrm{R}} \rho_{\mathrm{R}, n}^{\mathrm{L}}}
\end{aligned}
$$

Finally, the quantities $\rho_{\mathrm{R}, n}^{\mathrm{L}}, \tau_{n}^{\mathrm{L}}, \rho_{\mathrm{L}, n}^{\mathrm{R}}$, and $\tau_{n}^{\mathrm{R}}$ in Eq. 21 are recursively calculated in terms of the propagation matrix elements of the individual layers. Considering the left substructure in Fig. 3 with $J_{+, 0}^{\mathrm{h}}=0$, it is

$$
\begin{aligned}
\rho_{\mathrm{R}, n}^{\mathrm{L}} & =\frac{J_{+, n}^{\mathrm{h}}}{J_{-, n}^{\mathrm{h}}} \\
\tau_{n}^{\mathrm{L}} & =\frac{J_{-, 0}^{\mathrm{h}}}{J_{-, n}^{\mathrm{h}}}
\end{aligned}
$$


From the equations,

$$
\begin{aligned}
& J_{+, n}^{\mathrm{h}}=P_{11}^{(n)} J_{+, n-1}^{\mathrm{h}}+P_{12}^{(n)} J_{-, n-1}^{\mathrm{h}} \\
& J_{-, n}^{\mathrm{h}}=P_{21}^{(n)} J_{+, n-1}^{\mathrm{h}}+P_{22}^{(n)} J_{-, n-1}^{\mathrm{h}}
\end{aligned}
$$

and with $\operatorname{det} \mathbf{P}^{(n)}=1$, one gets the recursions,

$$
\begin{aligned}
\rho_{\mathrm{R}, 1}^{\mathrm{L}} & =\frac{P_{12}^{(1)}}{P_{22}^{(1)}} \\
\rho_{\mathrm{R}, n}^{\mathrm{L}} & =\frac{P_{11}^{(n)} \rho_{\mathrm{R}, n-1}^{\mathrm{L}}+P_{12}^{(n)}}{P_{21}^{(n)} \rho_{\mathrm{R}, n-1}^{\mathrm{L}}+P_{22}^{(n)}} \text { for } n=2,3, \ldots, N
\end{aligned}
$$

and

$$
\begin{aligned}
\tau_{1}^{\mathrm{L}} & =\frac{1}{P_{22}^{(1)}} \\
\tau_{n}^{\mathrm{L}} & =\frac{\tau_{n-1}^{\mathrm{L}}}{P_{21}^{(n)} \rho_{\mathrm{R}, n-1}^{\mathrm{L}}+P_{22}^{(n)}} \quad \text { for } n=2,3, \ldots, N .
\end{aligned}
$$

Similarly, considering the right substructure with $J_{-, N}^{\mathrm{h}}=0$, it is

$$
\begin{aligned}
\rho_{\mathrm{L}, n}^{\mathrm{R}} & =\frac{J_{-, n}^{\mathrm{h}}}{J_{+, n}^{\mathrm{h}}} \\
\tau_{\mathrm{n}}^{\mathrm{R}} & =\frac{J_{+, N}^{\mathrm{h}}}{J_{+, n}^{\mathrm{h}}}
\end{aligned}
$$

and the equations,

$$
\begin{aligned}
& J_{+, n}^{\mathrm{h}}=P_{22}^{(n+1)} J_{+, n+1}^{\mathrm{h}}-P_{12}^{(n+1)} J_{-, n+1}^{\mathrm{h}} \\
& J_{-, n}^{\mathrm{h}}=-P_{21}^{(n+1)} J_{+, n+1}^{\mathrm{h}}+P_{11}^{(n+1)} J_{-, n+1}^{\mathrm{h}}
\end{aligned}
$$

yield the recursions,

$$
\begin{aligned}
\rho_{\mathrm{L}, N-1}^{\mathrm{R}} & =\frac{P_{21}^{(N)}}{P_{22}^{(N)}} \\
\rho_{\mathrm{L}, n}^{\mathrm{R}} & =\frac{P_{11}^{(n+1)} \rho_{\mathrm{L}, n+1}^{\mathrm{R}}-P_{21}^{(n+1)}}{-P_{12}^{(n+1)} \rho_{\mathrm{L}, n+1}^{\mathrm{R}}+P_{22}^{(n+1)}} \text { for } n=N-2, \ldots 1,0
\end{aligned}
$$


and

$$
\begin{aligned}
\tau_{N-1}^{\mathrm{R}} & =\frac{1}{P_{22}^{(N)}} \\
\tau_{n}^{\mathrm{R}} & =\frac{\tau_{n+1}^{\mathrm{R}}}{-P_{12}^{(n+1)} \rho_{L, n+1}^{\mathrm{R}}+P_{22}^{(n+1)}} \text { for } n=N-2, \ldots .1,0
\end{aligned}
$$

By definition, it is $\mathbf{L}_{N}=\mathbf{R}_{0}$ and the transmissivity and the reflectivities at the left and right sides of the whole slab are

$$
\begin{aligned}
\tau & =\tau_{N}^{\mathrm{L}}=\tau_{0}^{\mathrm{R}} \\
\rho_{0} & =\rho_{\mathrm{L}, 0}^{\mathrm{R}} \\
\rho_{N} & =\rho_{\mathrm{R}, N}^{\mathrm{L}}
\end{aligned}
$$

\subsubsection{Boundary Conditions}

The values for $J_{+, 0}^{\mathrm{h}}$ and $J_{-, N}^{\mathrm{h}}$ in Eq. 21 are derived from the boundary conditions of the total radiative field, $J_{+, 0}$ and $J_{-, N}$ taking into account that $J_{ \pm}=J_{ \pm}^{\text {si }}+J_{ \pm}^{\mathrm{h}}$ and $J_{ \pm}^{\mathrm{si}}=\frac{1}{2}\left(F^{\mathrm{si}} \pm J^{\mathrm{si}}\right)$.

On the front surface, the slab is irradiated by a laser pulse of power flow $E(t)$. The slab internally conducts heat, but there is no heat flow at the front surface because of the vacuum surroundings. From $J_{Q}(0, t)=0$, it follows that $\frac{\partial T}{\partial x}(0, t)=0$, and thus from Eq. $5 b$, also $J^{\mathrm{si}}(0, t)=J_{0}^{\mathrm{si}}=0$. The boundary condition at the front surface is, therefore, given by

$$
J_{+, 0}=E(t)+\sigma_{\mathrm{B}} T_{\text {surr }}^{4}=J_{+, 0}^{\mathrm{h}}+\frac{1}{2}\left(F_{0}^{\mathrm{si}}+J_{0}^{\mathrm{si}}\right)=J_{+, 0}^{\mathrm{h}}+\sigma_{\mathrm{B}} T_{0}^{4}
$$

where Eq. $5 \mathrm{a}$ has been used and $T_{\text {surr }}$ denotes the surrounding temperature. The result is

$$
J_{+, 0}^{\mathrm{h}}=E-\sigma_{\mathrm{B}}\left(T_{0}^{4}-T_{\text {surr }}^{4}\right)
$$

At the rear surface, the slab is kept at constant temperature $T_{N}$ by a thermostat having reflectivity $\rho_{\text {sh }}$. The radiative balance at the rear surface is, thus, given by

$$
J_{-, N}=\rho_{\mathrm{sh}} J_{+, N}+\left(1-\rho_{\mathrm{sh}}\right) \sigma_{\mathrm{B}} T_{N}^{4}
$$

which yields

$$
J_{-, N}^{\mathrm{h}}+\sigma_{\mathrm{B}} T_{N}^{4}-\frac{1}{2} J_{N}^{\mathrm{si}}=\rho_{\mathrm{sh}}\left(J_{+, N}^{\mathrm{h}}+\sigma_{\mathrm{B}} T_{N}^{4}+\frac{1}{2} J_{N}^{\mathrm{si}}\right)-\left(1-\rho_{\mathrm{sh}}\right) \sigma_{\mathrm{B}} T_{N}^{4}
$$


and, as a consequence,

$$
J_{-, N}^{\mathrm{h}}-\rho_{\mathrm{sh}} J_{+, N}^{\mathrm{h}}=\frac{1}{2}\left(1+\rho_{\mathrm{sh}}\right) J_{N}^{\mathrm{si}}
$$

with

$$
J_{N}^{\mathrm{si}}=-\frac{8 \sigma_{\mathrm{B}} T^{3}}{A+2 S} \frac{\left(T_{N}-T_{N-1}\right)}{\delta_{N}}
$$

Substituting the flow $J_{+, N}^{\mathrm{h}}$ using Eq. $10 \mathrm{~b}$ finally gives

$$
J_{-, N}^{\mathrm{h}}=\frac{\left(\frac{1}{2}\left(1+\rho_{\mathrm{sh}}\right) J_{N}^{\mathrm{si}}+\rho_{\mathrm{sh}} \tau J_{+, 0}^{\mathrm{h}}\right)}{1-\rho_{\mathrm{sh}} \rho_{N}}
$$

Equations 16, 17, 18, 21, 39, and 42 represent the complete system of equations for the problem of transient energy transfer of the physical system defined above. The relatively complicated treatment of the solution of the homogeneous two-flux equations is necessary in order to be able to deal with temperature-dependent optical parameters.

\subsection{Numerical Solution Procedure}

The task remains to solve numerically the system, Eq. 16, of nonlinear, first-order differential equations. In order to get a compact notation, the quantities $D_{n}, \eta_{n}$, and the source terms $q_{n}$ are defined as follows:

$$
\begin{aligned}
D_{n} & =\frac{1}{\left(d c_{p}\right)_{n} \delta_{n}} \text { for } n=1,2, \ldots, N-1 \\
\eta_{n} & =\frac{2 k_{\mathrm{hrt}, n} k_{\mathrm{hrt}, n+1}}{\delta_{n+1} k_{\mathrm{hrt}, n}+\delta_{n} k_{\mathrm{hrt}, n+1}} \text { for } n=1,2, \ldots, N-1 \\
q_{n} & =D_{n} \Delta J_{n}^{\mathrm{h}} \text { for } n=1,2, \ldots, N-2
\end{aligned}
$$

with $\Delta J_{n}^{\mathrm{h}}$ given by Eq. 18. For the first layer, Eq. 16 is expressed as

$$
\frac{\mathrm{d} T_{1}}{\mathrm{~d} t}=-D_{1} \eta_{1} T_{1}+D_{1} \eta_{1} T_{2}+q_{1}
$$

where $T_{1}$ corresponds to the temperature of the irradiated surface denoted by $T_{0}$ previously. For the inner layers, it is

$\frac{\mathrm{d} T_{n}}{\mathrm{~d} t}=D_{n} \eta_{n-1} T_{n-1}-D_{n}\left(\eta_{n}+\eta_{n-1}\right) T_{n}+D_{n} \eta_{n} T_{n+1}+q_{n}$ for $n=2, \ldots, N-2$ 
Since the temperature $T_{N}$ of the boundary layer $n=N$ is kept constant, Eq. $43 \mathrm{~b}$ must be modified for the layer $N-1$ yielding

$$
\frac{\mathrm{d} T_{N-1}}{\mathrm{~d} t}=D_{N-1} \eta_{N-2} T_{N-2}-D_{N-1}\left(\eta_{N-1}+\eta_{N-2}\right) T_{N-1}+q_{N-1}
$$

with the source term,

$$
q_{N-1}=D_{N-1} \eta_{N-1} T_{N}+D_{N-1} \Delta J_{N-1}^{\mathrm{h}}
$$

The structure of the Eqs. $43 \mathrm{a}-\mathrm{c}$ becomes evident if a vector and matrix notation is used;

$$
\frac{\mathrm{d} \vec{T}}{\mathrm{~d} t}=\mathbf{H} \vec{T}+\vec{q}
$$

where $\vec{T}(x)=\left(T_{1}, T_{2}, \ldots, T_{N-1}\right)^{\mathrm{T}}, \vec{q}(x)=\left(q_{1}, q_{2}, \ldots, q_{N-1}\right)^{\mathrm{T}}$, and $\mathbf{H}$ is a tridiagonal $(N-1) \times(N-1)$ matrix

$$
\left(\begin{array}{ccccccc}
-D_{1} \eta_{1} & D_{1} \eta_{1} & 0 & \ldots & 0 & 0 & 0 \\
D_{2} \eta_{1} & -D_{2}\left(\eta_{1}+\eta_{2}\right) & D_{2} \eta_{2} & \ldots & 0 & 0 & 0 \\
\vdots & \vdots & \vdots & \vdots & \vdots & \vdots & \vdots \\
0 & 0 & 0 & \ldots & D_{N-2} \eta_{N-3} & -D_{N-2}\left(\eta_{N-3}+\eta_{N-2}\right) & D_{N-2} \eta_{N-2} \\
0 & 0 & 0 & \ldots & 0 & D_{N-1} \eta_{N-2} & -D_{N-1}\left(\eta_{N-2}+\eta_{N-1}\right)
\end{array}\right)
$$

Each element of $\mathbf{H}$ and of $\vec{q}$ depends on temperature and, thus, on time.

The transient temperature distribution in the material is now calculated with Eq. 44 performing an iterative time integration scheme. For a time increment $\Delta t_{m}=$ $t_{m+1}-t_{m}$, a Taylor expansion yields

$$
\vec{T}_{m+1}=\vec{T}_{m}+\left(\mathbf{H}_{m} \vec{T}_{m}+\vec{q}_{m}\right) \Delta t_{m}+O\left(\left(\Delta t_{m}\right)^{2}\right)
$$

with $\vec{T}_{m}=\vec{T}\left(t_{m}\right)$, etc. This formula is equivalent to

$$
\left(\mathbf{1}-\Delta t_{m} \mathbf{H}_{m}\right) \vec{T}_{m+1}=\vec{T}_{m}+\Delta t_{m} \vec{q}_{m}+O\left(\left(\Delta t_{m}\right)^{2}\right)
$$

the implicit differencing scheme being used for its superior numerical stability [17].

\subsection{Pyrometric Temperature Measurements}

The temperature measured by a one-color pyrometer, focusing on the front surface of the slab, can be obtained from the flux of radiant energy leaving the front surface $J_{-, 0}$. Inserting the value of $J_{-, N}^{\mathrm{h}}$ given by Eq. 42 into Eq. 10a calculated at cell $n=0$ yields

$$
J_{-, 0}^{\mathrm{h}}=\rho_{\mathrm{tot}} J_{+, 0}^{\mathrm{h}}+\frac{\tau}{2} \frac{\left(1+\rho_{\mathrm{sh}}\right)}{\left(1-\rho_{\mathrm{sh}} \rho_{N}\right)} J_{N}^{\mathrm{si}}
$$


where

$$
\rho_{\mathrm{tot}}=\rho_{0}+\frac{\tau^{2} \rho_{\mathrm{sh}}}{1-\rho_{\mathrm{sh}} \rho_{N}}
$$

is the reflectivity of the combined layer and thermostat system.

From Eq. 39 and $J_{0}^{\text {si }}=0$, it follows that

$$
J_{-, 0}=J_{-, 0}^{\mathrm{h}}+\sigma_{\mathrm{B}} T_{0}^{4}=\rho_{\mathrm{tot}}\left(E+\sigma_{\mathrm{B}} T_{\mathrm{surr}}^{4}\right)+\frac{\tau}{2} \frac{\left(1+\rho_{\mathrm{sh}}\right)}{\left(1-\rho_{\mathrm{sh}} \rho_{N}\right)} J_{N}^{\mathrm{si}}+\left(1-\rho_{\mathrm{tot}}\right) \sigma_{\mathrm{B}} T_{0}^{4}
$$

On the other hand, it follows from the definition of the temperature $T_{\text {pyr }}$ measured by the pyrometer on a surface with emissivity $\left(1-\rho_{\text {tot }}\right)$ that

$$
J_{-, 0}=\rho_{\text {tot }}\left(E+\sigma_{\mathrm{B}} T_{\text {surr }}^{4}\right)+\left(1-\rho_{\text {tot }}\right) \sigma_{\mathrm{B}} T_{\text {pyr }}^{4}
$$

Finally, we get from Eqs. 47 and 48 that

$$
T_{\mathrm{pyr}}^{4}=T_{0}^{4}+\frac{1}{\left(1-\rho_{\mathrm{tot}}\right) \sigma_{\mathrm{B}}} \frac{\tau\left(1+\rho_{\mathrm{sh}}\right)}{\left(1-\rho_{\mathrm{sh}} \rho_{N}\right)} J_{N}^{\mathrm{si}} \geq T_{0}^{4}
$$

since $J_{N}^{\mathrm{si}} \geq 0$ for the given problem according to Eq. 41 .

It follows that the pyrometer measures an upper bound of the true surface temperature $T_{0}$. For a thick slab, however, $\tau \rightarrow 0$ and $T_{\text {pyr }} \rightarrow T_{0}$. We conclude that the temperature measured with a one-color pyrometer using the actual value of the transient emissivity is the actual true value of the transient surface temperature for opaque samples. This is not obvious because there may be a considerable temperature interval within the optical escape depth with a distinct influence on the actual value of the reflectivity and emissivity if the optical material properties depend on temperature (see Figs. 12, 14 and Table 2). For transparent samples considerable deviations may occur, as shown in Sect. 3, since the reflectivity properties of the thermostat matter in this case.

\section{Simulation: Results}

The algorithm developed in Sect. 2 was implemented in a computer code, and simulations of the energy transfer were performed for yttria-stabilized zirconia, an example of a typical technical STM used at very high temperatures. For calculations, the medium is assumed to have a constant density of $5.6 \times 10^{3} \mathrm{~kg} \cdot \mathrm{m}^{-3}$ and temperature-dependent thermophysical properties as listed in Table 1 . The temperature dependence of the absorption coefficient of zirconia is not well known for elevated temperatures. Cabannes and Billard [18] report the results of an investigation on cubic zirconia from room temperature to $1,930 \mathrm{~K}$ in the wavelength region from $3 \mu \mathrm{m}$ to $6 \mu \mathrm{m}$ where they demonstrate that the absorption coefficient increases monotonically. The increase is 
Table 1 Thermophysical properties used in the calculations

\begin{tabular}{llllll}
\hline$T(\mathrm{~K})$ & $k\left(\mathrm{~W} \cdot \mathrm{m}^{-1} \cdot \mathrm{K}^{-1}\right)$ & $\begin{array}{l}\mathrm{c}_{p}\left(\mathrm{~J} \cdot \mathrm{kg}^{-1} \cdot \mathrm{K}^{-1}\right) \\
{[22]}\end{array}$ & $\begin{array}{l}\rho^{\mathrm{a}}(-) \\
{[20]}\end{array}$ & $\begin{array}{l}A^{\mathrm{b}}\left(\mathrm{m}^{-1}\right) \\
S=1 \times 10^{4} \mathrm{~m}^{-1}\end{array}$ & $\begin{array}{l}A^{\mathrm{b}}\left(\mathrm{m}^{-1}\right) \\
S=5 \times 10^{4} \mathrm{~m}^{-1}\end{array}$ \\
\hline 300 & 1.40 & 456 & 0.65 & $9.42 \times 10^{2}$ & $4.71 \times 10^{3}$ \\
400 & 1.40 & 517 & 0.65 & $9.42 \times 10^{2}$ & $4.71 \times 10^{3}$ \\
500 & 1.40 & 549 & 0.65 & $9.42 \times 10^{2}$ & $4.71 \times 10^{3}$ \\
600 & 1.50 & 569 & 0.65 & $9.42 \times 10^{2}$ & $4.71 \times 10^{3}$ \\
700 & 1.50 & 596 & 0.65 & $9.42 \times 10^{2}$ & $4.71 \times 10^{3}$ \\
800 & 1.50 & 605 & 0.65 & $9.42 \times 10^{2}$ & $4.71 \times 10^{3}$ \\
900 & 1.50 & 614 & 0.65 & $9.42 \times 10^{2}$ & $4.71 \times 10^{3}$ \\
1000 & 1.50 & 622 & 0.648 & $9.56 \times 10^{2}$ & $4.78 \times 10^{3}$ \\
1100 & 1.50 & 630 & 0.64 & $1.01 \times 10^{3}$ & $5.06 \times 10^{3}$ \\
1200 & 1.50 & 637 & 0.625 & $1.13 \times 10^{3}$ & $5.63 \times 10^{3}$ \\
1300 & 1.50 & 644 & 0.6 & $1.33 \times 10^{3}$ & $6.67 \times 10^{3}$ \\
1400 & 1.50 & 644 & 0.56 & $1.73 \times 10^{3}$ & $8.64 \times 10^{3}$ \\
1500 & 1.50 & 644 & 0.49 & $2.65 \times 10^{3}$ & $1.33 \times 10^{4}$ \\
1600 & 1.50 & 644 & 0.4 & $4.50 \times 10^{3}$ & $2.25 \times 10^{4}$ \\
1700 & 1.50 & 644 & 0.33 & $6.80 \times 10^{3}$ & $3.40 \times 10^{4}$ \\
1800 & 1.50 & 644 & 0.28 & $9.26 \times 10^{3}$ & $4.63 \times 10^{4}$ \\
1900 & 1.50 & 644 & 0.23 & $1.29 \times 10^{4}$ & $6.44 \times 10^{4}$ \\
2000 & 1.50 & 644 & 0.2 & $1.60 \times 10^{4}$ & $8.00 \times 10^{4}$ \\
2100 & 1.50 & 644 & 0.18 & $1.87 \times 10^{4}$ & $9.34 \times 10^{4}$ \\
2200 & 1.50 & 644 & 0.17 & $2.03 \times 10^{4}$ & $1.01 \times 10^{5}$ \\
2300 & 1.50 & 644 & 0.16 & $2.21 \times 10^{4}$ & $1.10 \times 10^{5}$ \\
2400 & 1.50 & 644 & 0.155 & $2.30 \times 10^{4}$ & $1.15 \times 10^{5}$ \\
2500 & 1.50 & 644 & 0.15 & $2.41 \times 10^{4}$ & $1.20 \times 10^{5}$ \\
2600 & 1.50 & 644 & 0.15 & $2.41 \times 10^{4}$ & $1.20 \times 10^{5}$ \\
2700 & 1.50 & 644 & 0.15 & $2.41 \times 10^{4}$ & $1.20 \times 10^{5}$ \\
2800 & 1.50 & 644 & 0.15 & $2.41 \times 10^{4}$ & $1.20 \times 10^{5}$ \\
\hline
\end{tabular}

${ }^{a}$ Reflectivity at $0.63 \mu \mathrm{m}$

${ }^{\mathrm{b}}$ Calculated with Eq. 50

almost linear up to $1,600 \mathrm{~K}$ but much more pronounced above this temperature. However, they do not propose any mathematical or empirical relation that would allow extrapolation of their results to the temperature and wavelength range considered in this work. For this reason, the values for the absorption coefficient were derived here on the basis of diffuse reflectivity measurements of an infinitely thick sample. From the relation between the reflectivity $\rho$ and the quotient $A / S$ of the absorption and scattering coefficients,

$$
\frac{\left(1-\rho^{2}\right)}{2 \rho}=\frac{A}{S},
$$

which is valid for the two-flux approximation of an isothermal infinitely thick slab with constant material parameters, it follows that the temperature dependence of the reflectivity determines the temperature dependence of the quotient $A / S$. Since the scattering coefficient depends largely on porosity and typical grain size, it can be assumed that it does not change with temperature as long as no structural changes 
Table 2 Optical penetration depth for zirconia

\begin{tabular}{|c|c|c|}
\hline$T(\mathrm{~K})$ & $\begin{array}{l}1 / W(\mathrm{~m}) \\
S=1 \times 10^{4} \mathrm{~m}^{-1}\end{array}$ & $\begin{array}{l}1 / W(\mathrm{~m}) \\
S=5 \times 10^{4} \mathrm{~m}^{-1}\end{array}$ \\
\hline 300 & $2.25 \times 10^{-4}$ & $4.50 \times 10^{-5}$ \\
\hline 400 & $2.25 \times 10^{-4}$ & $4.50 \times 10^{-5}$ \\
\hline 500 & $2.25 \times 10^{-4}$ & $4.50 \times 10^{-5}$ \\
\hline 600 & $2.25 \times 10^{-4}$ & $4.50 \times 10^{-5}$ \\
\hline 700 & $2.25 \times 10^{-4}$ & $4.50 \times 10^{-5}$ \\
\hline 800 & $2.25 \times 10^{-4}$ & $4.50 \times 10^{-5}$ \\
\hline 900 & $2.25 \times 10^{-4}$ & $4.50 \times 10^{-5}$ \\
\hline 1000 & $2.23 \times 10^{-4}$ & $4.47 \times 10^{-5}$ \\
\hline 1100 & $2.17 \times 10^{-4}$ & $4.34 \times 10^{-5}$ \\
\hline 1200 & $2.05 \times 10^{-4}$ & $4.10 \times 10^{-5}$ \\
\hline 1300 & $1.88 \times 10^{-4}$ & $3.75 \times 10^{-5}$ \\
\hline 1400 & $1.63 \times 10^{-4}$ & $3.26 \times 10^{-5}$ \\
\hline 1500 & $1.29 \times 10^{-4}$ & $2.58 \times 10^{-5}$ \\
\hline 1600 & $9.52 \times 10^{-5}$ & $1.90 \times 10^{-5}$ \\
\hline 1700 & $7.41 \times 10^{-5}$ & $1.48 \times 10^{-5}$ \\
\hline 1800 & $6.08 \times 10^{-5}$ & $1.22 \times 10^{-5}$ \\
\hline 1900 & $4.86 \times 10^{-5}$ & $9.71 \times 10^{-6}$ \\
\hline 2000 & $4.17 \times 10^{-5}$ & $8.33 \times 10^{-6}$ \\
\hline 2100 & $3.72 \times 10^{-5}$ & $7.44 \times 10^{-6}$ \\
\hline 2200 & $3.50 \times 10^{-5}$ & $7.00 \times 10^{-6}$ \\
\hline 2300 & $3.28 \times 10^{-5}$ & $6.57 \times 10^{-6}$ \\
\hline 2400 & $3.18 \times 10^{-5}$ & $6.35 \times 10^{-6}$ \\
\hline 2500 & $3.07 \times 10^{-5}$ & $6.14 \times 10^{-6}$ \\
\hline 2600 & $3.07 \times 10^{-5}$ & $6.14 \times 10^{-6}$ \\
\hline 2700 & $3.07 \times 10^{-5}$ & $6.14 \times 10^{-6}$ \\
\hline 2800 & $3.07 \times 10^{-5}$ & $6.14 \times 10^{-6}$ \\
\hline
\end{tabular}

occur. Therefore, the temperature dependence of $A$ is given by the temperature dependence of the reflectivity through Eq. 50.

Based on the work of Makino et al. [19], the two values, $S=1 \times 10^{4} \mathrm{~m}^{-1}$ and $S=5 \times 10^{4} \mathrm{~m}^{-1}$, were chosen for the scattering coefficient. Since the model developed so far cannot deal with wavelength-dependent optical properties, we restricted the analysis to one characteristic wavelength, and we used the absorption coefficients at $0.63 \mu \mathrm{m}$ calculated from the reflectivity data of Petrov et al. [20] with Eq. 50 (see Table 1). In Table 2, the parameter $1 / W$, which is a measure of the optical penetration and escape depth of the material, is listed as a function of temperature for the two chosen values of the scattering coefficient.

In the following sections, the influence of various experimental parameters on the temperature distribution in the sample, on the reflectivity, and on the pyrometric temperature is studied. In particular, we investigate the effects of the optical thickness, of the reflectivity of the thermostat (which is significant when the layer is semitransparent), of the laser pulse time, and of the rear and initial temperatures of the sample. 


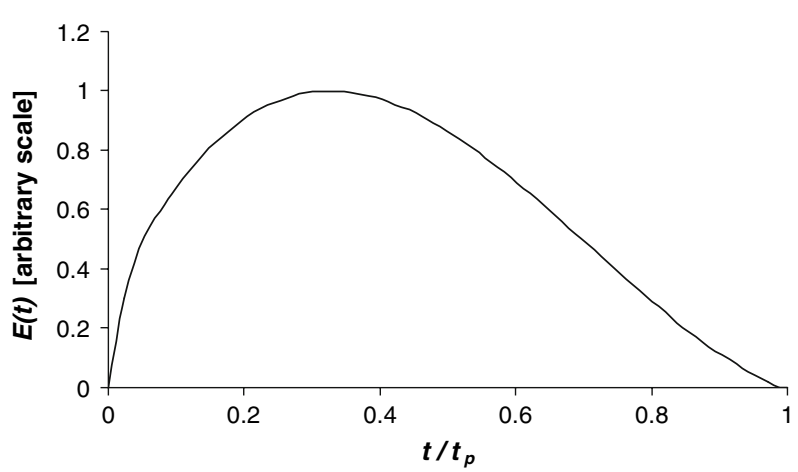

Fig. 4 Power profile of the laser pulse used for the numerical calculations. $t_{\mathrm{p}}$ denotes the total length of the laser pulse. The area under the curve is proportional to the total energy flow $E_{\text {tot }}$ of the pulse

In the following, the initial temperature of the layer is assumed to be $300 \mathrm{~K}$ and in thermal equilibrium with the surroundings except when otherwise stated. The thermostat ${ }^{1}$ keeping the rear surface at constant temperature $T_{N}=300 \mathrm{~K}$ is supposed to have a reflectivity $\rho_{\mathrm{sh}}=0.65$. The layer material has a scattering coefficient of $S=1 \times 10^{4} \mathrm{~m}^{-1}$. A laser pulse of a standardized shape,

$$
E(t)=E_{\mathrm{tot}} f\left(\frac{t}{t_{p}}\right)
$$

given in Fig. 4 is used for the calculations. The duration of the pulse $t_{\mathrm{p}}=5 \times$ $10^{-3} \mathrm{~s}$, except when stated differently. The calculations were always performed with an equally spaced grid of 2,000 points. A total transient time of $60 \mathrm{~s}$ was investigated with a time grid consisting of 17,000 points, the spacing being denser for the heating phase than for the cooling phase. In the figures, only representative time points are shown. Since the energy required to reach the desired surface temperature strongly varies with the material properties and boundary conditions, calculations were performed with different energy inputs as reported in the text.

\subsection{Influence of Thickness}

To investigate the influence of the layer thickness on the thermal profile, a set of different thicknesses $(L=2,1,0.5,0.1 \mathrm{~mm})$ was considered. All calculations reported in this section refer to a total input laser energy flow $E_{\text {tot }}=7.28 \times 10^{6} \mathrm{~J} \cdot \mathrm{m}^{-2}$.

As an example of an opaque sample, the temperature profiles in the layer of $2 \mathrm{~mm}$ are shown in Fig. 5 as a function of time. During heating (see Fig. 5a), the temperature of the layer progressively increases reaching its maximum when all the laser power is delivered. During cooling (see Fig. 5b), the value of the surface temperature mono-

\footnotetext{
1 It should be noted that the thermostat could also be viewed as the sample holder or the substrate of a coating where the zirconia layer represents the coating.
} 

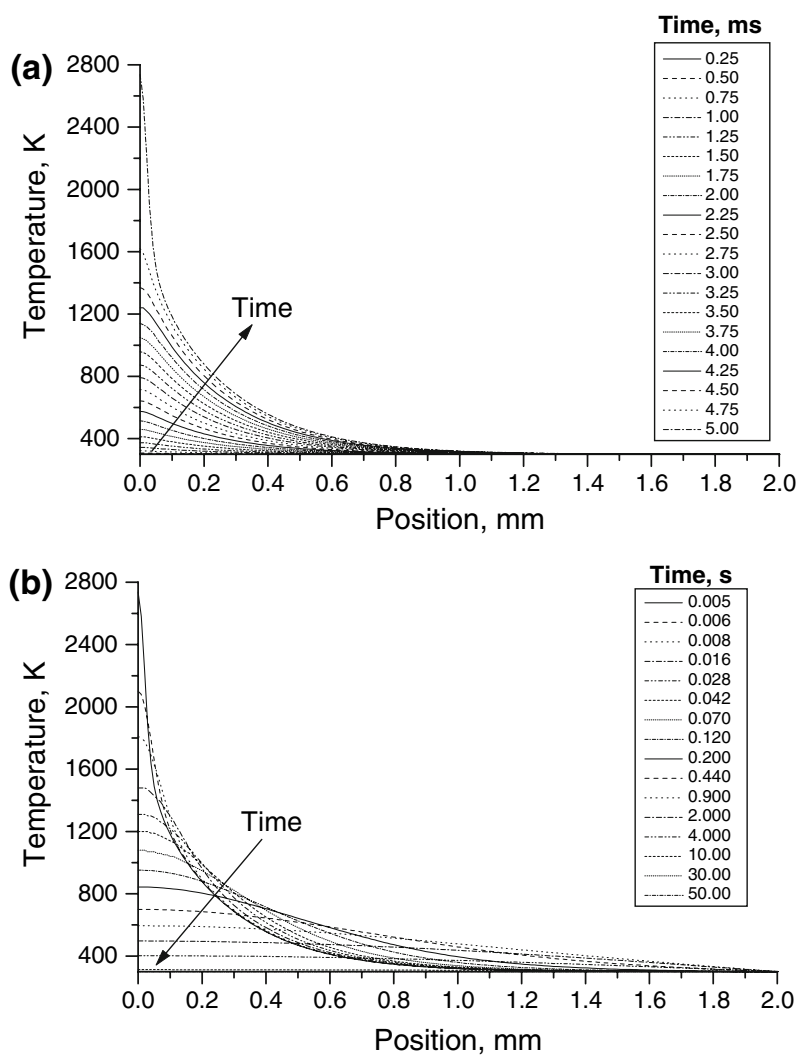

Fig. 5 Transient temperature distribution for a sample with $L=2 \mathrm{~mm}, S=1 \times 10^{4} \mathrm{~m}^{-1}, T_{N}=300 \mathrm{~K}$, $\rho_{\mathrm{sh}}=0.65$, and $t_{\mathrm{p}}=5 \times 10^{-3} \mathrm{~s}$ : (a) heating phase during the laser pulse and (b) cooling phase after the laser has been switched off

tonically decreases. In the interior, however, the temperature still increases for some time due to the heat redistribution in the layer.

It should be noted that the spatial temperature distribution decreases monotonically also in the cooling phase, even though the direction of the total energy flow at the surface is now opposite to the one for the heating phase. Radiative cooling distinctly differs in this respect from cooling by heat conduction. In our model, the value of the temperature gradient at the surfaces is determined uniquely by the boundary conditions for the temperature field. In the calculations presented, the sample is assumed to be in vacuum with no heat flow across the irradiated surface yielding always a zero value of the temperature gradient. Conductive or convective heat flow across the irradiated surface can also be modeled leading to a positive value of the temperature gradient near the surface in the heating phase as well as in the cooling phase as long as the surface temperature exceeds the temperature of the surroundings. In this case, the spatial maximum of the temperature would be found in the interior of the sample and not at its surface. 

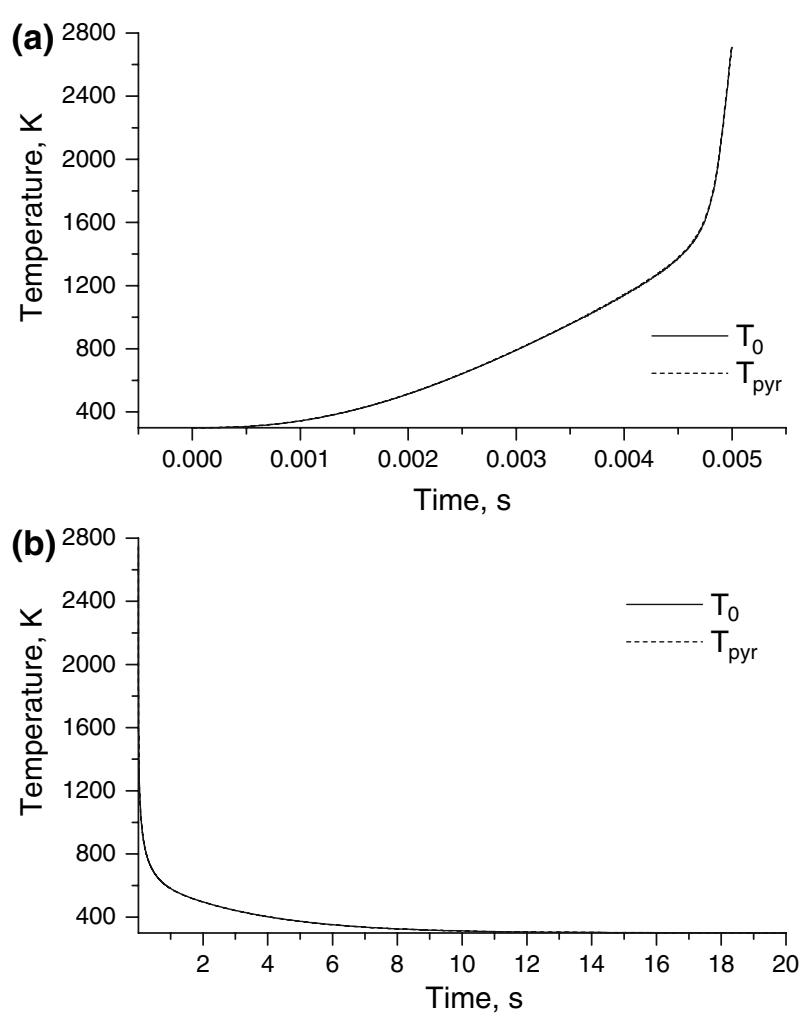

Fig. 6 Transient temperature of the front surface for a sample with $L=2 \mathrm{~mm}, S=1 \times 10^{4} \mathrm{~m}^{-1}$, $T_{N}=300 \mathrm{~K}, \rho_{\mathrm{sh}}=0.65$, and $t_{\mathrm{p}}=5 \times 10^{-3} \mathrm{~s}$ : (a) heating phase during the laser pulse and (b) cooling phase after the laser has been switched off. The surface temperature $T_{0}$ and the temperature $T_{\text {pyr }}$ measured with a pyrometer coincide

In Fig. 6, the temperature at the front surface is shown as a function of time. When the laser is switched off at $t_{p}=0.005 \mathrm{~s}$, the material slowly cools, and after about $13 \mathrm{~s}$, the temperature reaches the surrounding value of $300 \mathrm{~K}$. The sample is opaque, and the true temperature of the front surface coincides with the temperature that is measured by a pyrometer in accordance with the findings in Sect. 2.4.

In Fig. 7, curves 1 and 2, the isothermal reflectivity of the material and the calculated transient reflectivities during heating and cooling are represented. The transient reflectivities strongly differ from the isothermal values, the difference being more pronounced during heating than during cooling due to the more pronounced temperature drop within the layer optically accessible from the surface. The thickness of this layer, the optical penetration depth, is of the order of $1 / W$. At the maximum surface temperature reached, $1 / W$ is equal to $3.07 \times 10^{-5} \mathrm{~m}$ and the temperature drops from $2,737 \mathrm{~K}$ to $1,890 \mathrm{~K}$ within the distance $1 / \mathrm{W}$ from the surface. Therefore, an appreciable part of the light reflected at the surface stems from a region with a low quotient of $A / S$ leading to a reflection coefficient well above its isothermal value for the surface temperature. 


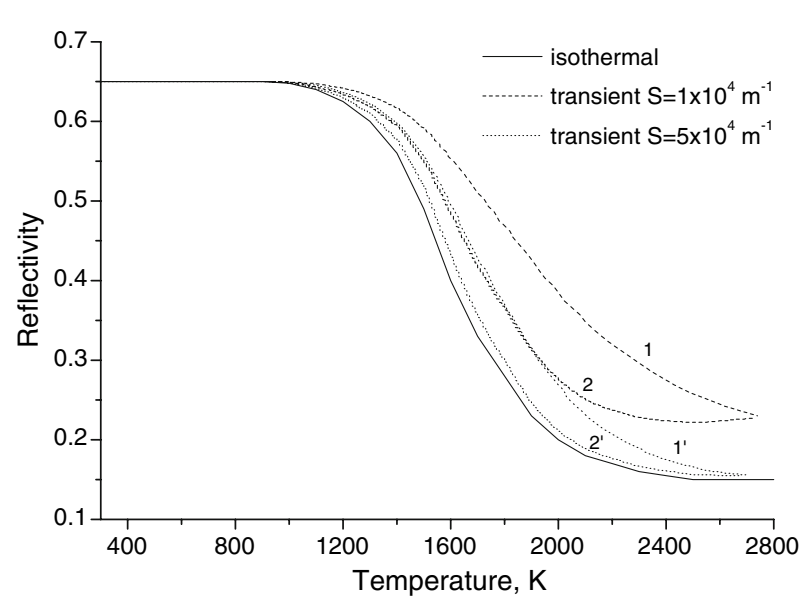

Fig. 7 Isothermal and transient reflectivities as a function of temperature for a sample with $L=2 \mathrm{~mm}$, $T_{N}=300 \mathrm{~K}, \rho_{\mathrm{sh}}=0.65, t_{\mathrm{p}}=5 \times 10^{-3} \mathrm{~s}, S=1 \times 10^{4} \mathrm{~m}^{-1}$, and $S=5 \times 10^{4} \mathrm{~m}^{-1}$. Branches $1,1^{\prime}$ show the transient reflectivity during heating, branches $2,2^{\prime}$ during cooling

The samples with thicknesses of 1 and $0.5 \mathrm{~mm}$ are still opaque. The temperature profiles and the transient reflectivity values show the same behavior as in the case of the $2 \mathrm{~mm}$ thick layer. However, the picture drastically changes if its thickness is further reduced. As an example, the results for a thickness of $0.1 \mathrm{~mm}$ are reported in Fig. 8. The maximum surface temperature reached is much lower than in the previous cases, and the sample is semitransparent for temperatures below 1,500 K. As soon as the sample becomes semitransparent, the temperature that a pyrometer measures using the correct emissivity value (one minus the actual transient reflectivity) starts to deviate from the true surface temperature (see Fig. 9). The pyrometric temperature is then always higher than the true surface temperature in accordance with the theoretical findings in Sect. 2.4, Eq. 49. In Fig. 10, curves 1 and 2, the corresponding transient reflectivity values are reported.

\subsection{Influence of the Reflectivity of the Sample Holder}

The influence of the reflectivity of the thermostat on the temperature profiles and on the reflectivity of the sample was investigated for the thickness $L=2,1,0.5$, and $0.1 \mathrm{~mm}$. As expected, there is no such influence for the opaque samples. However, there is a drastic influence in the case of the semitransparent sample with $L=0.1 \mathrm{~mm}$. With a value of $\rho_{\mathrm{sh}}=0.2$ instead of $\rho_{\mathrm{sh}}=0.65$ and an identical energy input $\left(E_{\text {tot }}=7.28 \times 10^{6} \mathrm{~J} \cdot \mathrm{m}^{-2}\right)$, a much lower temperature is reached $(1,333 \mathrm{~K}$ instead of $2,140 \mathrm{~K}$ ) due to the smaller radiative flux reflected by the thermostat and the increase of absorptivity between $1,300 \mathrm{~K}$ and $2,000 \mathrm{~K}$ (see Table 1 ). The influence of the reflectivity of the thermostat on the calculated transient reflectivity values is illustrated in Fig. 10. Curves $1^{\prime}$ and $2^{\prime}$ referring to $\rho_{\mathrm{sh}}=0.2$ indicate indeed a much lower reflectivity value compared with the values calculated for $\rho_{\text {sh }}=0.65$. 

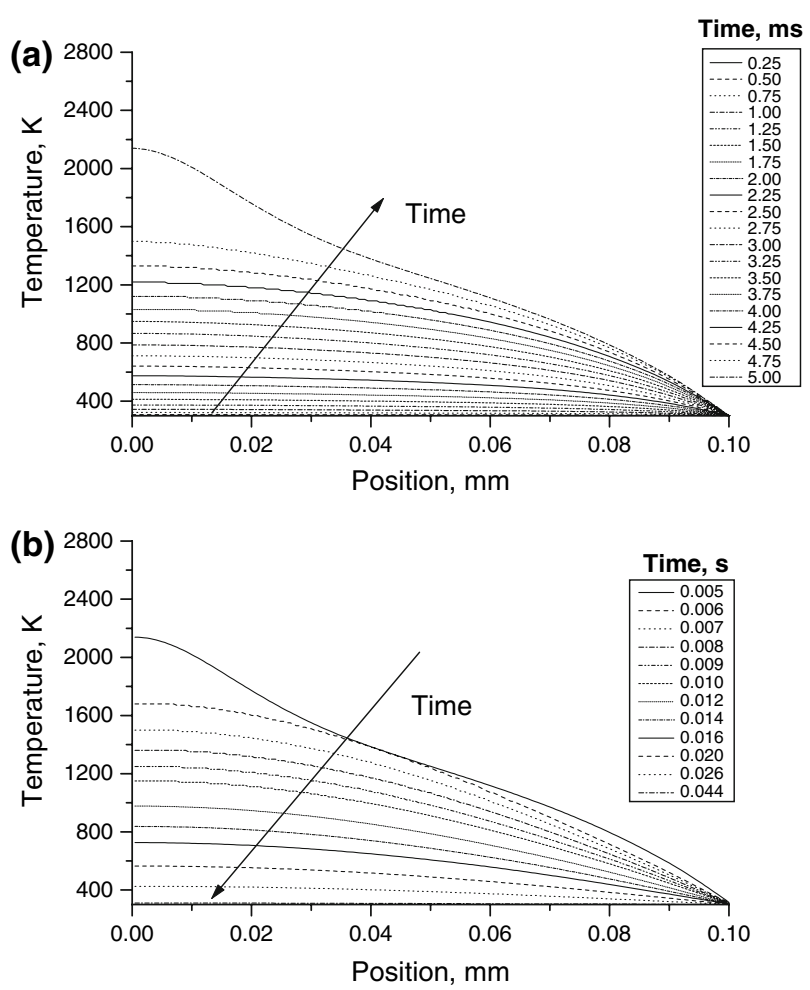

Fig. 8 Transient temperature distribution for a sample with $L=0.1 \mathrm{~mm}, S=1 \times 10^{4} \mathrm{~m}^{-1}, T_{N}=300 \mathrm{~K}$, $\rho_{\mathrm{sh}}=0.65$, and $t_{\mathrm{p}}=5 \times 10^{-3} \mathrm{~s}$ : (a) heating phase during the laser pulse and (b) cooling phase after the laser has been switched off

\subsection{Influence of the Scattering Coefficient}

The influence of the scattering coefficient was investigated for thicknesses of 2, 1, 0.5, 0.1 , and $0.05 \mathrm{~mm}$. The results obtained with $S=5 \times 10^{4} \mathrm{~m}^{-1}$ show that the sample is opaque for the first four studied thicknesses, implying $T_{\mathrm{pyr}}=T_{0}$ and semitransparent for $L=0.05 \mathrm{~mm}$. As expected, the temperature profiles in the samples were much steeper than for the case of $S=1 \times 10^{4} \mathrm{~m}^{-1}$ because of the higher scattering coefficient. In order to obtain the same temperature increase as before, considerably less energy was required $\left(E_{\mathrm{tot}}=2.2 \times 10^{6} \mathrm{~J} \cdot \mathrm{m}^{-2}\right.$ instead of $7.28 \times 10^{6} \mathrm{~J} \cdot \mathrm{m}^{-2}$ for the $2 \mathrm{~mm}$ layer).

The calculated reflectivities for $L=2 \mathrm{~mm}$ are reported in Fig. 7, curves $1^{\prime}$ and $2^{\prime}$. The comparison of the results obtained for $S=1 \times 10^{4} \mathrm{~m}^{-1}$ and $S=5 \times 10^{4} \mathrm{~m}^{-1}$ shows that the higher scattering coefficient results in calculated transient reflectivities that are closer to the isothermal values.

\subsection{Influence of the Laser Pulse Time}

The influence of the laser pulse time has been investigated for a sample of thickness $L=2 \mathrm{~mm}$. In Fig. 11, the temperature profiles for laser pulse times of $t_{p}=5 \times 10^{-4} \mathrm{~s}$ 

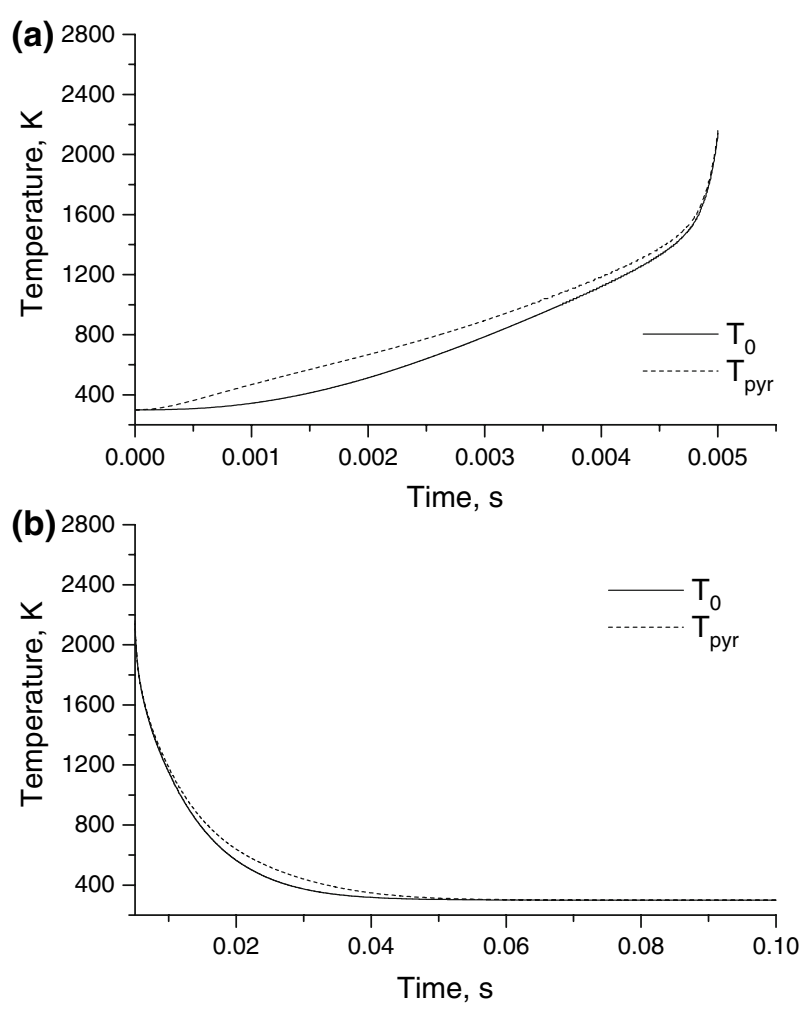

Fig. 9 Transient temperature at the front surface for a sample with $L=0.1 \mathrm{~mm}, S=1 \times 10^{4} \mathrm{~m}^{-1}$, $T_{N}=300 \mathrm{~K}, \rho_{\mathrm{sh}}=0.65$, and $t_{\mathrm{p}}=5 \times 10^{-3} \mathrm{~s}$ : (a) heating phase during the laser pulse and (b) cooling phase after the laser has been switched off. There are substantial deviations of the temperature $T_{\text {pyr }}$ measured with a pyrometer from the true surface temperature $T_{0}$

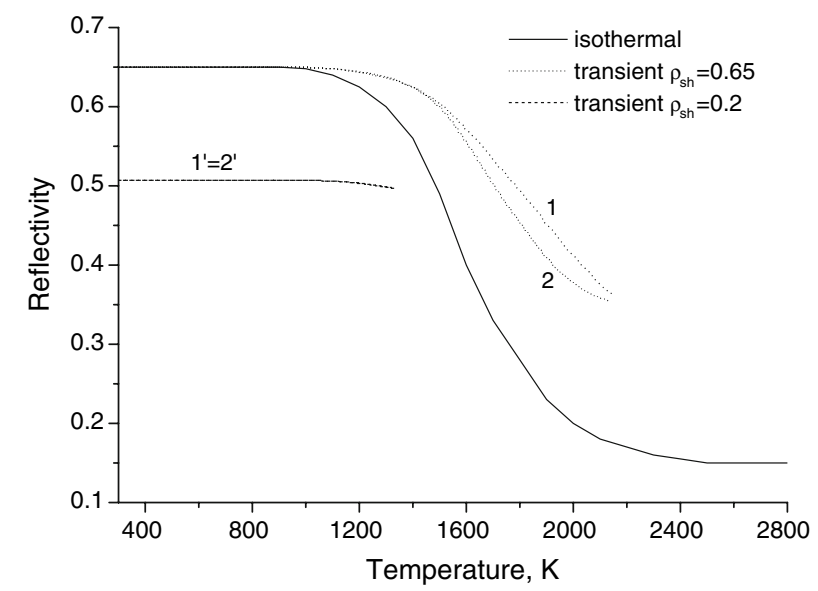

Fig. 10 Isothermal and transient reflectivities as a function of temperature for a sample with $L=0.1 \mathrm{~mm}$, $S=1 \times 10^{4} \mathrm{~m}^{-1}, T_{N}=300 \mathrm{~K}, \rho_{\mathrm{sh}}=0.65$ and $\rho_{\mathrm{sh}}=0.2$, and $t_{\mathrm{p}}=5 \times 10^{-3} \mathrm{~s}$. Branches $1,1^{\prime}$ show the reflectivity during heating, branches $2,2^{\prime}$ during cooling 

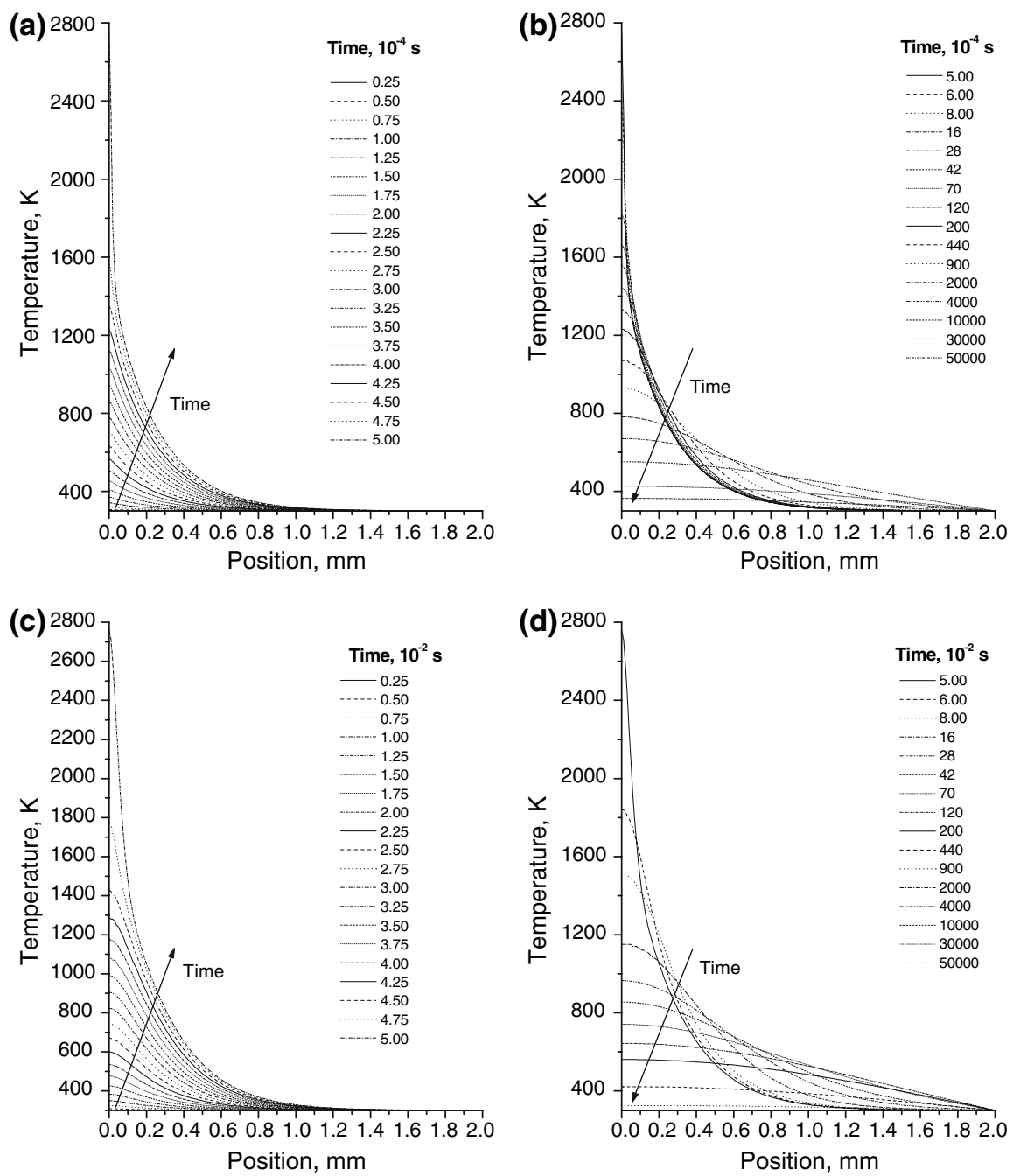

Fig. 11 Transient temperature distribution for a sample with $L=2 \mathrm{~mm}, S=1 \times 10^{4} \mathrm{~m}^{-1}, T_{N}=300 \mathrm{~K}$, and $\rho_{\mathrm{sh}}=0.65$ for different total lengths $t_{\mathrm{p}}$ of the laser pulse: (a) heating during the laser pulse, $t_{\mathrm{p}}=$ $5 \times 10^{-4} \mathrm{~s}$, (b) cooling after the laser has been switched off, $t_{\mathrm{p}}=5 \times 10^{-4} \mathrm{~s}$, (c) heating during the laser pulse, $t_{\mathrm{p}}=5 \times 10^{-2} \mathrm{~s}$, and (d) cooling after the laser has been switched off, $t_{\mathrm{p}}=5 \times 10^{-2} \mathrm{~s}$

with $E_{\mathrm{tot}}=6.64 \times 10^{6} \mathrm{~J} \cdot \mathrm{m}^{-2}$ and of $t_{p}=5 \times 10^{-2} \mathrm{~s}$ with $E_{\mathrm{tot}}=9.35 \times 10^{6} \mathrm{~J} \cdot \mathrm{m}^{-2}$ are presented.

Comparing these results with those obtained for $t_{p}=5 \times 10^{-3} \mathrm{~s}$, which are reported in Fig. 5, it can be seen that the pulse duration strongly influences the temperature profile within the sample. The temperature gradients within the optical escape depths of $3.07 \times 10^{-5} \mathrm{~m}$ for the maximum temperature of about $2,760 \mathrm{~K}$ are shown in Fig. 12 for all the three cases studied. As expected, the longer the laser pulse, the smaller is the thermal gradient within the optical escape depth. The same pattern is observed 


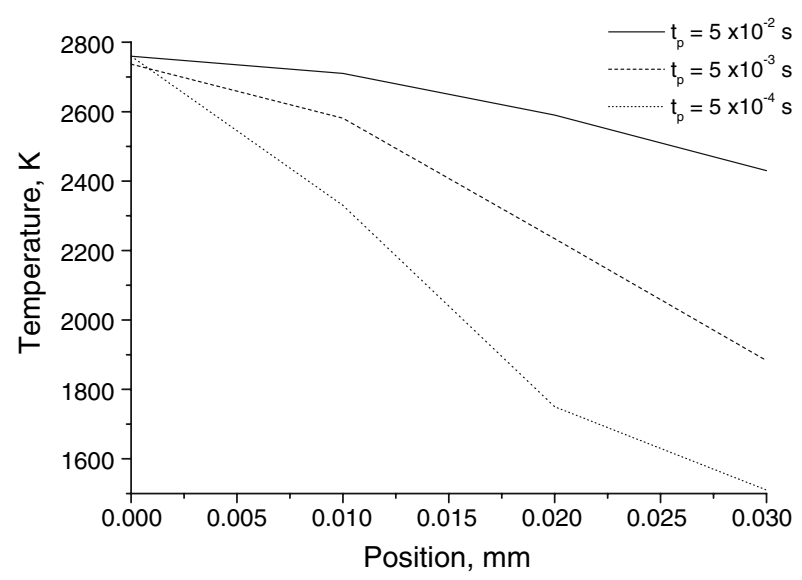

Fig. 12 Temperature distribution near the surface at the maximum temperature reached just before the laser is switched off for a sample with $L=2 \mathrm{~mm}, S=1 \times 10^{4} \mathrm{~m}^{-1}, T_{N}=300 \mathrm{~K}$, and $\rho_{\mathrm{sh}}=0.65$. The total length of the laser pulse $t_{\mathrm{p}}$ is varied, and the total energy flow of the laser is adapted to get approximately equal values of the maximal surface temperature

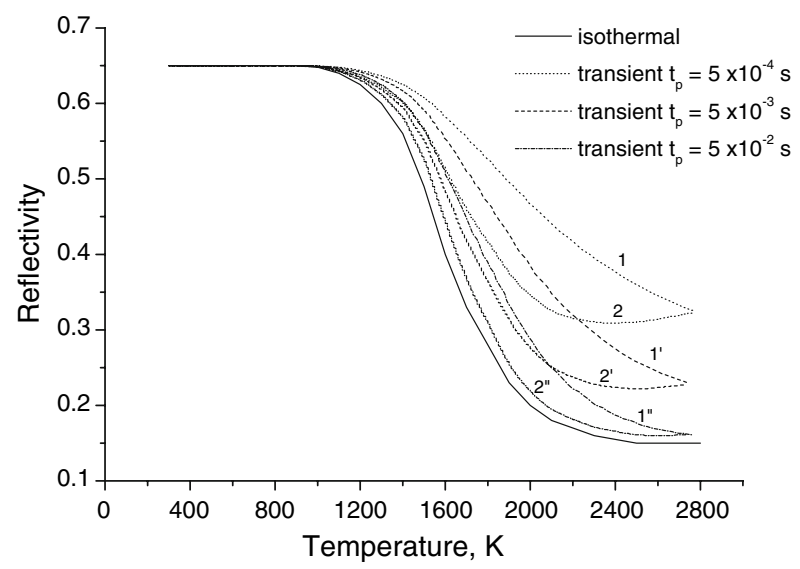

Fig. 13 Isothermal and transient reflectivities as a function of temperature for different total lengths $t_{\mathrm{p}}$ of the laser pulse and for a sample with $L=2 \mathrm{~mm}, S=1 \times 10^{4} \mathrm{~m}^{-1}, T_{N}=300 \mathrm{~K}$, and $\rho_{\mathrm{sh}}=0.65$. Branches $1,1^{\prime}, 1^{\prime \prime}$ show the reflectivity during heating, branches $2,2^{\prime}, 2^{\prime \prime}$ during cooling

for the transient reflectivities: the longer the laser pulse, the closer the values of the calculated reflectivity to the isothermal values (see Fig. 13).

\subsection{Influence of the Initial and Rear Temperatures}

The influence of the initial temperature on the thermal response of the sample was investigated for the sample of thickness $L=2 \mathrm{~mm}$. Since the aim of this work is to investigate the thermal response of an isothermal sample to a laser shot, the constant rear temperature of the slab was always assumed to be equal to its initial temperature: 


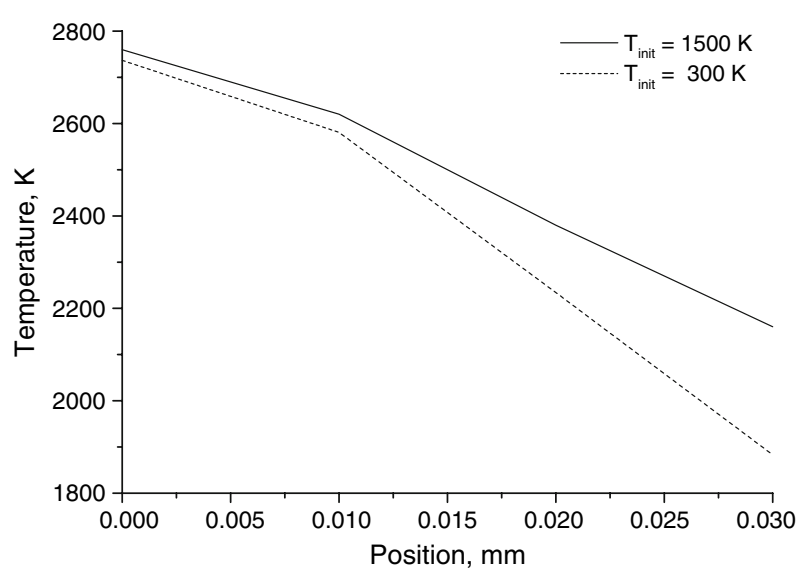

Fig. 14 Temperature distribution near the surface at the maximum temperature reached just before the laser is switched off for two different initial temperatures $T_{\text {init }}$ of a sample with $L=2 \mathrm{~mm}, S=1 \times 10^{4} \mathrm{~m}^{-1}$, $\rho_{\mathrm{sh}}=0.65$, and $t_{\mathrm{p}}=5 \times 10^{-3} \mathrm{~s}$. The temperature of the thermostat is always equal to the initial temperature of the sample

$T_{N}=T_{\text {init }}$. The input energy was chosen such that the sample is heated to the same maximum surface temperature as for the case of $T_{\text {init }}=300 \mathrm{~K}$, necessitating a reduced energy input of $E_{\mathrm{tot}}=5.6 \times 10^{5} \mathrm{~J} \cdot \mathrm{m}^{-2}$ for $T_{\text {init }}=1,500 \mathrm{~K}$. A comparison of the results for this higher initial temperature with the results obtained for an initial temperature of $300 \mathrm{~K}$ reveals that the temperature profile in the interior of the samples remains qualitatively the same but with a reduced thermal gradient (see Fig. 14). With $T_{\text {init }}=1,500 \mathrm{~K}$, the lower temperature gradient within the optical escape depth of the sample influences the values of the transient reflectivity which are much closer to the isothermal ones than in the case of $T_{\text {init }}=300 \mathrm{~K}$ (see Fig. 15).

\section{Discussion and Conclusion}

Semitransparent materials are frequently used in high-temperature applications where combined heat conduction and radiative transport play an important role. As a consequence, there is considerable interest in the measured values of the thermophysical and optical properties of such materials at high temperatures. Radiative properties such as absorption and scattering coefficients are preferably determined from reflectivity and transmissivity measurements on isothermal samples. At very high temperatures, measurements under isothermal conditions may, however, become impracticable and one has to rely on transient measurements, e.g., using laser pulses to heat the sample surface to the desired temperatures.

The interpretation of transient reflectivity and transmission data in terms of optical properties of the material such as the absorption and scattering coefficients of the two-flux model for radiation is, however, not straightforward since semitransparent materials typically combine low thermal conductivity with low values of absorption over an extended wavelength region, and the material parameters often depend on 


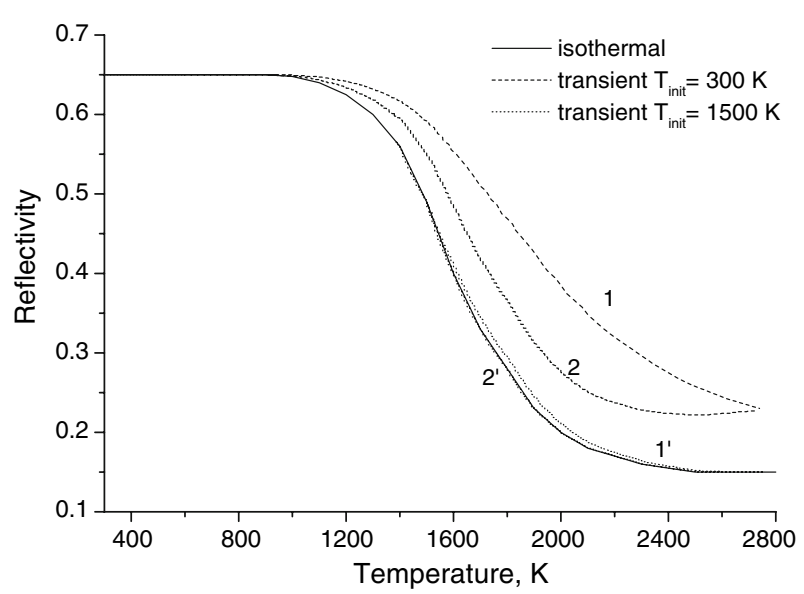

Fig. 15 Isothermal and transient reflectivities as a function of temperature for different initial temperatures and for a sample with $L=2 \mathrm{~mm}, S=1 \times 10^{4} \mathrm{~m}^{-1}, \rho_{\mathrm{sh}}=0.65$, and $t_{\mathrm{p}}=5 \times 10^{-3} \mathrm{~s}$. The temperature of the thermostat is always equal to the initial temperature of the sample. Branches $1,1^{\prime}$ show the reflectivity during heating, branches $2,2^{\prime}$ during cooling

temperature. In a transient heating or cooling regime, considerable temperature differences may occur within the optical penetration depth, and the incident light used for a reflectivity measurement may simultaneously probe regions with different absorption and scattering properties. The responses from these different regions then contribute to the transient value of the reflectivity.

As a consequence, the measured transient values of the reflectivity to the same surface temperature may considerably differ in the heating and cooling phases of a laser-heated sample as reported by Petrov et al. [20]. We fully agree with the conclusion of the authors "that the reflectivity and emissivity under intense laser heating are not physical constants which may be related to some temperature, but depend on the temperature field in the surface layer of the sample." This implies that such reflectivity data can be related to the optical material properties only by modeling the combined heat and radiative transport in the sample, taking into account the experimental circumstances such as, for instance, the proper boundary conditions for temperature and radiation.

Such a model is presented here for a gray slab with temperature-dependent thermophysical and radiative properties. Although the conditions of our calculations are not strictly identical with the experimental conditions for the reflectivity measurements reported in [20], the same qualitative results are obtained: the reflectivity values measured with laser-heated samples differ considerably for the heating and cooling phases and both values are different from the isothermal values. These deviations strongly increase with increasing power of the heating laser (compare Fig. 7 in [20] with Fig. 7 of the present work). The differences in the hypothetical temperature distributions reported by the authors of [20] and the ones reported in this work (compare Fig. 5 of [20] with our Figs. 5b, 8b, 11b, 11d) are due to the different experimental conditions. In the experiments carried out in [20], the irradiated front surface is cooled by 
convection and melting of the material occurs (in our calculations, the samples are placed in vacuum and melting is not considered).

The calculations in this work are based on a model for a gray slab and disregard phase transitions. Phase transitions can, however, be easily taken into account by choosing the specific heat as a function of temperature $c_{p}(T)$ accordingly. In addition, the model can be extended to cover the wavelength dependence of radiative properties by dividing the total wavelength region into intervals where the absorption and scattering coefficients are constant with respect to wavelength. This will improve the modeling of semitransparent materials which typically exhibit an absorption edge and often are heated and probed with radiation of different wavelengths.

Acknowledgement This work has been supported by the Swiss Federal Office of Energy (BFE).

\section{Nomenclature}

A Absorption coefficient $\left(\mathrm{m}^{-1}\right)$

$c_{p} \quad$ Specific heat, $\left(\mathrm{J} \cdot \mathrm{kg}^{-1} \cdot \mathrm{K}^{-1}\right)$

$d \quad$ Density $\left(\mathrm{kg} \cdot \mathrm{m}^{-3}\right)$

$E_{\text {tot }} \quad$ Total energy flow of laser pulse $\left(\mathrm{J} \cdot \mathrm{m}^{-2}\right)$

$E(t) \quad$ Laser power flow $\left(\mathrm{W} \cdot \mathrm{m}^{-2}\right)$

$F \quad J_{+}+J_{-}$, auxiliary flux quantity $\left(\mathrm{W} \cdot \mathrm{m}^{-2}\right)$

$F^{\mathrm{h}} \quad$ Solution of the homogeneous two-flux equations $\left(\mathrm{W} \cdot \mathrm{m}^{-2}\right)$

$F^{\mathrm{si}} \quad$ Solution of the in-homogeneous two-flux equations $\left(\mathrm{W} \cdot \mathrm{m}^{-2}\right)$

$J_{+}, J_{-} \quad$ Radiative fluxes in the positive and negative $x$-directions $\left(\mathrm{W} \cdot \mathrm{m}^{-2}\right)$

$J^{\text {h }} \quad$ Flux quantity, solution of the homogeneous two-flux equations $\left(\mathrm{W} \cdot \mathrm{m}^{-2}\right)$

$J^{\text {si }} \quad$ Flux quantity, solution of the inhomogeneous two-flux equations $\left(\mathrm{W} \cdot \mathrm{m}^{-2}\right)$

$J_{Q} \quad$ Conductive heat flux $\left(\mathrm{W} \cdot \mathrm{m}^{-2}\right)$

$J \quad$ Total radiative flux $\left(\mathrm{W} \cdot \mathrm{m}^{-2}\right)$

$k \quad$ Thermal conductivity $\left(\mathrm{W} \cdot \mathrm{m}^{-1} \cdot \mathrm{K}^{-1}\right)$

$k_{\mathrm{hrt}} \quad$ Combined heat and radiative transfer coefficient $\left(\mathrm{W} \cdot \mathrm{m}^{-1} \cdot \mathrm{K}^{-1}\right)$

$L \quad$ Thickness of the slab (m)

$\mathbf{L}_{n} \quad$ Transfer matrix for left substructure at boundary $n(-)$

$\mathbf{P} \quad$ Transfer matrix for homogeneous two-flux equations (-)

$\mathbf{P}^{(n)} \quad$ Transfer matrix of layer $n(-)$

$\mathbf{R}_{n} \quad$ Transfer matrix of right substructure at boundary $n(-)$

$S \quad$ Scattering coefficient $\left(\mathrm{m}^{-1}\right)$

$t \quad$ Time (s)

$t_{p} \quad$ Duration of the laser pulse (s)

$T \quad$ Temperature $(\mathrm{K})$

$T_{N} \quad$ Temperature of thermostat (K)

$T_{\mathrm{pyr}} \quad$ Temperature measured with a pyrometer $(\mathrm{K})$

$T_{\text {surr }} \quad$ Temperature of surroundings (K)

$\Delta t_{m} \quad t_{m}-t_{m-1}$, step for time integration (s)

$W^{-1} \quad$ Optical penetration depth of the medium (m) 


$\begin{array}{ll}\delta_{n} x_{n}-x_{n-1}, & \text { space grid step at point } n, 1 \leq n \leq N(\mathrm{~m}) \\ \varepsilon_{\mathrm{L} / \mathrm{R}} & \text { Emissivity at the left/right surface of a layer }(-) \\ \rho_{\mathrm{L} / \mathrm{R}} & \text { Reflectivity at the left/right surface of a layer }(-) \\ \rho_{0} & \text { Reflectivity at the front surface of the slab }(-) \\ \rho_{N} & \text { Reflectivity at the rear surface of the slab }(-) \\ \rho_{\mathrm{R}, n}^{\mathrm{L}} & \text { Reflectivity of the left substructure at the right side of cell } n(-) \\ \rho_{\mathrm{L}, n}^{\mathrm{R}} & \text { Reflectivity of the right substructure at the left side of cell } n(-) \\ \rho_{\mathrm{sh}} & \text { Reflectivity of the thermostat }(-) \\ \rho_{\mathrm{tot}} & \text { Transient reflectivity of the combined system (slab and } \\ & \text { thermostat) }(-) \\ \sigma_{B} & \text { Stefan-Boltzmann constant }\left(\mathrm{W} \cdot \mathrm{m}^{-2} \cdot \mathrm{K}^{-4}\right) \\ \tau_{\mathrm{L} / \mathrm{R}} & \text { Transmissivity at the left/right surface of a layer }(-) \\ \tau_{\delta} & \text { Transmissivity of a layer of thickness } \delta(-) \\ \tau_{n}^{\mathrm{L} / \mathrm{R}} & \text { Transmissivity of the left/right substructure }(-) \\ \tau & \text { Transmissivity of the slab }(-)\end{array}$

\section{References}

1. R. Siegel, Int. J. Eng. Sci. 36, 1701 (1998)

2. R. Siegel, J. Heat Transfer 120, 4 (1998)

3. R. Siegel, J. Thermophys. 6, 77 (1992)

4. L.H. Liu, J. Quant. Spectrosc. Radiat. Transfer 74, 97(2002)

5. S. Andre, A. Degiovanni, Int. J. Heat Mass Transfer 38, 3401 (1995)

6. T. Heping, B. Mestre, M. Lallemand, J. Heat Transfer 113, 166 (1991)

7. H. Tan, L. Ruan, T.W. Tong, Int. J. Heat Mass Transfer 43, 311 (2000)

8. L.H. Liu, H.P. Tan, T.W. Tong, Int. J. Heat Mass Transfer 44, 3335 (2001)

9. L.K. Matthews, R. Viskanta, F.P. Incropera, J. Solar Energy Eng. 107, 29 (1985)

10. O. Hahn, F. Raether, M.C. Arduini-Schuster, J. Fricke, Int. J. Heat Mass Transfer 40, 689 (1997)

11. H. Tan, L. Ruan, X. Xia, Q. Yu, T.W. Tong, Int. J. Heat Mass Transfer 42, 2967 (1999)

12. S. Andre, A. Degiovanni, J. Heat Transfer 120, 943 (1998)

13. M. Musella, H.R. Tschudi, Int. J. Thermophys. 26, 981 (2005)

14. R. Siegel, J.R. Howell, Thermal Radiation Heat Transfer, 4th edn. (Taylor \& Francis, New York, 2002), pp. 625-662

15. P. Kubelka, F. Munk, Zeit. für Techn. Physik 12, 593 (1931)

16. R.G. Deissler, J. Heat Transfer 86, 240 (1964)

17. W.H. Press, S.A. Teukolsky, W.T. Vetterling, B.P. Flannery, Numerical Recepies in C, 2nd edn. (Cambridge University Press, 1996), pp. 734-747

18. F. Cabannes, D. Billard, Int. J. Thermophys. 8, 97 (1987)

19. T. Makino, T. Kunimoto, I. Sakai, H. Kinoshita, Heat Transfer Jpn. Res. 13, 33 (1984)

20. V.A. Petrov, A.Y. Vorobyev, A.P. Chernyshev, High Temp. High Press. 34, 657 (2002)

21. V.Y. Checkhovskoy, A.M. Banaev, Heat and Mass Transfer, vol. VII, ed. by A.V. Likov, Nauka I Tekhnika (URSS, Minsk 1968), p. 591

22. J.P. Coughlin, E.G. King, J. Am. Chem. Soc. 72, 2262 (1950) 